\title{
ChilV3 Acts as a Novel Target of WRKY40 to Mediate Pepper Immunity Against Ralstonia solanacearum Infection
}

\author{
Zhiqin Liu, ${ }^{1,2}$ Lanping Shi, ${ }^{1,2}$ Yahong Weng, ${ }^{1,2}$ Huasong Zou, ${ }^{3}$ Xia Li, ${ }^{1,2}$ Sheng Yang, ${ }^{1,2}$ Shanshan Qiu, ${ }^{1,2}$ \\ Xueying Huang, ${ }^{1,2}$ Jinfeng Huang, ${ }^{1,2}$ Ansar Hussain,, ${ }^{1,2}$ Kan Zhang, ${ }^{1,2}$ Deyi Guan, ${ }^{1,2}$ and Shuilin He ${ }^{1,2,+}$ \\ ${ }^{1}$ Ministry of Education Key Laboratory of Plant Genetic Improvement and Comprehensive Utilization, Fujian Agriculture and \\ Forestry University, Fuzhou, 350002 China \\ ${ }^{2}$ College of Crop Science, Fujian Agriculture and Forestry University \\ ${ }^{3}$ College of Plant Protection, Fujian Agriculture and Forestry University, Fuzhou, 350002 China
}

Accepted 15 March 2019.

ChiIV3, a chitinase of pepper (Capsicum annuum), stimulates cell death in pepper plants. However, there are only scarce reports on its role in resistance against bacterial wilt disease such as that caused by Ralstonia solanacearum and their transcriptional regulation. In this study, the silencing of ChiIV3 in pepper plants significantly reduced the resistance to $R$. solanacearum. The transcript of ChilV3 was induced by $R$. solanacearum inoculation (RSI) as well as exogenous application of methyl jasmonate and abscisic acid. The bioinformatics analysis revealed that the ChiIV3 promoter consists of multiple stress-related cis elements, including six W-boxes and one MYB1AT. With the 5' deletion assay in the ChilV3 promoter, the W4-box located from -640 to -635 bp was identified as the cis element that is required for the response to RSI. In addition, the W4-box element was shown to be essential for the binding of the ChiIV3 promoter by the WRKY40 transcription factor, which is known to positively regulate the defense response to $R$. solanacearum. Site-directed mutagenesis in the W4-box sequence impaired the binding of WRKY40 to the ChilV3 promoter. Subsequently, the transcription of ChilV3 decreased in WRKY40-silenced pepper plants. These results demonstrated that the expression of the defense gene ChilV3 is controlled through multiple modes of regulation, and WRKY40 directly binds to the W4-box element of the ChiIV3 promoter region for its transcriptional regulation.

Keywords: plant responses to pathogens, pathogenesis-related proteins, resistance genes

Zhiqin Liu, Lanping Shi, and Yahong Weng are co-first authors.

${ }^{\dagger}$ Corresponding author: S. He; shlhe201304@aliyun.com

Funding: This work was supported by grants from the Natural Science Foundation of Fujian Province, China (2017J01436), National Natural Science Foundation of China (31501767, 31572136, and 31372061), and Young and Middle-aged Teachers Education Scientific Research Project of Fujian Provincial Department of Education (2016). The funding agencies had no role in the study design, data collection, data analysis, decision to publish, or preparation of the manuscript.

*The $\boldsymbol{e}$-Xtra logo stands for "electronic extra" and indicates that two supplementary tables are published online.

The author(s) declare no conflict of interest.

(c) 2019 The American Phytopathological Society
Host plants have developed their defense mechanisms to survive against microbial pathogens in natural environments (Jones and Dangl 2006; Yin et al. 2015; Zhong et al. 2018). The defense mechanisms comprised activation of the mitogenactivated protein kinase (MAPK) cascades and accumulation of reactive oxygen species and nitric oxide. A number of transcription factors, such as WRKY, bZIP, ERF, and NAC, have been involved in defense induction by regulating the expression of pathogenesis-related $(P R)$ genes (Hwang et al. 2011; Jones et al. 2016; Kim and Hwang 2011; Mur et al. 2017; Park et al. 2013; Tsuda et al. 2013; Zhang and Feng 2014; Zhou et al. 2017). Plants are dependent on innate immunity to fight against pathogen attack, due to the lack of the adaptive immune system (Jones and Dangl 2006). Plants have evolved two characteristic interconnecting layers of immunity, namely, pattern triggered immunity (PTI) and effector-triggered immunity (ETI) (Hein et al. 2009; Jones and Dangl 2006). Both systems of immunity have been reported to share common signaling components and, possibly, similar sets of $P R$ genes. In the PTI and ETI signaling pathways, $P R$ genes are involved; however, they have varied expression levels and resistance duration (Thomma et al. 2011; Tsuda and Katagiri 2010).

Chitin, a polymer of $\mathrm{N}$-acetyl-D-glucosamine, is a fungal cell-wall component that is not found in plant cells (Vanholme et al. 2014). Host plants containing chitin-degrading enzymes can destroy fungal cell walls and inhibit pathogen multiplication. Chitin fragments (chitooligosaccharides) may induce PTI reaction in host plants (Wan et al. 2008a and b). Therefore, chitins are recognized as a kind of pathogen-associated molecular pattern for a large number of fungal pathogens. Even though the bacterial cell wall does not contain chitin, some plant chitinases showed suppression effects on bacterial growth. For example, Brassica juncea chitinase BjCHI1 exhibited antimicrobial properties against both fungal and bacterial pathogens (Guan and Chye 2008). As chitinases are generally encoded by a gene family, they constitute a group of PR proteins involved in plant immunity (Ahmed et al. 2012; Iseli et al. 1996; Lee et al. 2012; Nakahara and Masuta 2014). The expression of chitinases is regulated in plants and has exhibited an inducible pattern at the transcription level upon pathogen infection (Gao et al. 2014; Hong and Hwang 2006). A vast array of cis elements in promoters is responsible for perceiving environmental stress signals or pathogen invasion (Baykal et al. 2006; Chen et al. 2015; Gao et al. 2014; Hong and Hwang 2006; Liu et al. 2017). Since each chitinase promoter 
harbors different cis elements, the transcription pattern of one single gene varies from the other genes. The transcriptional regulation of chitinase genes seems very complicated, a particular splicing pattern was recently found on a basic chitinase $P R 3 b$ gene in Burley tobacco that caused an alternative splicing in low-nicotine nicl and nic2 mutants and was regulated by jasmonate (JA) and ethylene (ET) (Tominaga et al. 2016). However, the transcription regulation and function of chitinase genes have not been fully explained, especially in nonmodel plants.

WRKY proteins represent one of the largest transcription factor (TF) families with one or two conserved WRKY domains that bind to W-box cis elements in the promoters of their targets (Agarwal et al. 2011; Eulgem 2006; Eulgem et al. 1999, 2000; Rushton et al. 2010, 2012). This specific regulation mode is not only essential for plant growth and development but also for response to environmental stressors, such as pathogen attack (Eulgem 2006; Eulgem and Somssich 2007; Ishihama and Yoshioka 2012; Pandey and Somssich 2009). In general, a set of WRKYs constitute a WRKY web within which one WRKY acts as a TF to regulate the transcription of other members or physically interacts with other members (Baldoni et al. 2013; Eulgem and Somssich 2007). Most WRKY TFs can arbitrarily bind to distinctive and conserved W-boxes [TTGAC $(\mathrm{C} / \mathrm{T})$ ]. Residues in the conserved WRKYGQK motif interact with DNA bases mainly through extensive apolar interactions with thymine methyl groups. The importance of these contacts was verified by substituting relevant $T$ bases with $U$ and surface plasmon resonance analyses of DNA binding (Yamasaki et al. 2005; Yamasaki et al. 2012). However, knowledge about the specific targets of a given WRKY TF for it to fulfill the specific function and how it could determine its target specificity is very limited.

Pepper (Capsicum annuum) is a vegetable of great economic significance worldwide that often suffered various soil-borne diseases, such as bacterial wilt caused by $R$. solanacearum and phytophthora blight (Choi and Hwang 2011; Choi et al. 2012, 2013; Fu et al. 2015; Liu et al. 2016; Römer et al. 2007; Tang et al. 2007; Wang et al. 2014). A better understanding of the plant immune system will help improve the disease resistance of pepper at a genetic level. Fifteen putative chitinase genes have been identified in the published genome of pepper (Kim et al. 2014b). Among the chitinase family, a basic class II chitinase, CAChi2 (Hong and Hwang 2006), and class IV chitinase, ChitIV (Kim et al. 2015), have been found to be involved in response to pathogen infection. Overexpression of CAChi2 in Arabidopsis plants has shown an increased resistance against Pseudomonas syringae pv. tomato and increased tolerance to $\mathrm{NaCl}$-induced osmotic stress (Hong and Hwang 2006). The class IV chitinase CaChitIV interacts with CaPIK1 and promotes the CaPIK1-triggered cell death and defense responses (Kim et al. 2015). However, the majority of these chitinases in pepper have not been functionally characterized so far.

Our previous study showed that WRKY40, a member of the WRKY subgroup IIa in pepper, is transcriptionally up-regulated by high temperature and high humidity or by $R$. solanacearum inoculation and it acts as a positive regulator in the response of pepper to $R$. solanacearum infection or in thermotolerance under high humidity (Dang et al. 2013). However, what defense genes targeted by WRKY40 and how they function remain to be elucidated. In the present study, we focused on the pepper ChiIV3, a cDNA-encoding class IV chitinase. The objective of this study was to uncover the role of ChiIV3 in pepper immunity during $R$. solanacearum infection and how it is transcriptionally regulated. Our data showed that ChiIV3 is transcriptionally regulated by WRKY40 via the W4-box within its promoter and contributes positively to the response of pepper to the attack of $R$. solanacearum.

\section{RESULTS}

ChilV3 is induced by $R$. solanacearum, methyl jasmonate (MeJA), and abscisic acid (ABA).

In our previous study, ChilV3, a cDNA encoding a chitinase, was identified in pepper plant (Liu et al. 2017). To understand its transcription regulation, a $1,017 \mathrm{bp}$ promoter sequence of ChilV3 was analyzed using the PlantCARE database. The results indicated that the ChiIV3 promoter consisted of numerous regulatory cis elements, including six W-boxes, three MYB1ATs, two HSEs (heat-shock elements), three MYB1ATs, and two CGTCA-motifs. An ERELEE4 motif and TATA-box were detected at the $5^{\prime}$ and $3^{\prime}$ termini, respectively (Fig. 1A). Since W-box, MYB1AT, and CGTCA-motifs are pathogenresponsive and hormone-associated cis elements, transcription of ChilV3 was investigated after RSI and treatment with phytohormones. Upon RSI, the transcript level of ChiIV3 was found to be significantly increased from 1 to $24 \mathrm{~h}$ postinoculation (hpi), with the highest level exhibited at 12 hpi (Fig. 1B). Transcript of ChiIV3 showed diverse changes in response to the phytohormone treatments. ChiIV3 transcripts were induced by exogenous application of MeJA and ABA and were reduced by application of ethephon (ETH), brassinolide (BR), and salicylic acid (SA) (Fig. 1C through G). These results demonstrated that the expression of ChiIV3 was regulated by RSI and phytohormones.

\section{ChiIV3-silencing pepper plants showed severe bacterial wilt symptoms.}

To ascertain the role of ChiIV3 in disease resistance, a loss-offunction of ChiIV3 was produced by virus-induced gene silencing (VIGS) (Fig. 2A). The transcript levels of ChiIV3 in roots and stems of silenced pepper plants were detected to assess the silencing efficiency. ChiIV3 transcripts in roots and stems of ChiIV3-silenced pepper plants were, respectively, approximately one fifth and one half of those in the wild-type pepper plants (unsilenced), indicating that the expression of ChilV3 was effectively inhibited (Fig. 2B). Trypan blue staining indicated that ChiIV3-silencing mitigated the hypersensitive cell death induced by $R$. solanacearum infection in pepper leaves (Fig. 2C). Predominantly, ChiIV3-silenced plants developed more susceptibility to $R$. solanacearum infection. On comparison with unsilenced control plants, such susceptibility was demonstrated by more severe disease symptoms (Fig. 2D), a higher relative disease index (Fig. 2E), and lower transcriptional levels of defenserelated marker genes, including jasmonic acid (JA)-dependent $D E F 1$ and ABA-responsive ABRI (Fig. 2F). These results suggest that silencing of ChiIV3 significantly reduced the resistance of pepper plants against $R$. solanacearum infection.

\section{Identification of ChiIV3 promoter regions essential for RSI and phytohormone induction.}

To determine which cis element was essential for perceiving signals of RSI, MeJA, or ABA, a 5' deletion assay was conducted on the ChilV3 promoter (Fig. 3A). The reporter systems linked with promoter deletions and plant transformation were successfully established in our previous study (Liu et al. 2017). $\beta$-glucuronidase (GUS) quantification data revealed that $\mathrm{p} 1017$, p891, and p712 deletions were sufficient to trigger GUS expression under RSI, while p459 and p276 deletions could not trigger GUS expression. This indicated that the cis elements between -712 bp and -459 bp might be the key domain required for RSI, comprising two CGTCA motifs, two MYB1ATs, and three noticeable W-boxes, i.e., W3-box $\left(\mathrm{W}^{-672}\right.$ to -667$)$, W4-box 
$\left(\mathrm{W}^{-640}\right.$ to -635$)$, and W5-box $\left(\mathrm{W}^{-466}\right.$ to $\left.{ }^{-461}\right)$ (Fig. 3B). The cis elements associated with MeJA and ABA were different from those associated with RSI. The region between -459 and $-276 \mathrm{bp}$ comprising the W6-box was found to be associated with MeJA (Fig. 3C), while the region between -891 and -712 bp comprising the W2-box and a MYB1AT motif was associated with ABA signaling (Fig. 3D).

\section{W4-box in the ChiIV3 promoter is critical for RSI.}

Three W-boxes were located from -712 bp to -459 bp in the ChilV3 promoter. To determine which $\mathrm{W}$-box was responsible for perceiving RSI, ChiIV3 promoters were constructed with a mutation in the W3-box, W4-box, or W5-box (Fig. 4A). Agrobacterium cells containing the ChilV3 promoter-GUS fusions and its mutants were infiltrated into the leaves of 8-weekold pepper plants. After $24 \mathrm{~h}$, agroinfiltrated leaves were inoculated with $R$. solanacearum. At $48 \mathrm{hpi}$, the inoculated leaves were tested for GUS activity. In comparison with the wild-type promoter, both W3-box and W5-box mutants showed similar induction effects with GUS activity (Fig. 4B), while the W4box mutant could not induce the expression of GUS triggered by $R$. solanacearum inoculation. This showed that the W4-box element was critical for ChiIV3 in response to $R$. solanacearum infection. In addition, the W6-box (W-box-339 to $-334 \mathrm{bp}$ ) located between -339 and -334 bp and MYB1AT element located between -735 and -730 bp were essential for the response of pepper plants to the exogenous application of MeJA and ABA (Fig. 5A and B).

\section{W4-box is required for WRKY40 binding} to the ChiIV3 promoter.

To clearly understand ChilV3 transcriptional regulation, a yeast one-hybrid system was performed to identify the putative upstream regulator involved in Chilv3 activation. As the
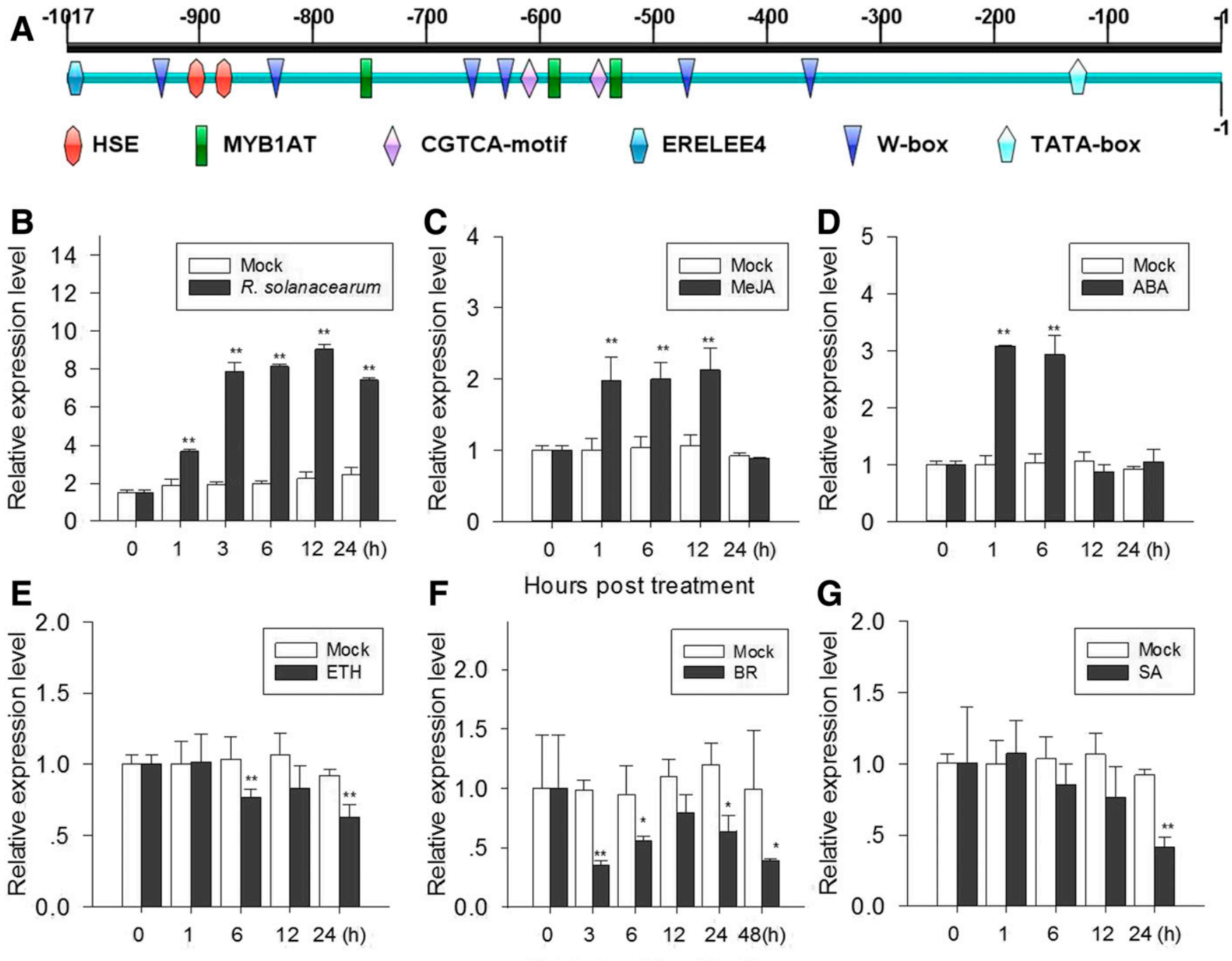

Hours post treatment

Fig. 1. Promoter and expression profile analysis of ChiIV3 in response to Ralstonia solanacearum inoculation and exogenous phytohormones. A, Potential stress-related cis-acting elements present in the promoter of ChiIV3 ( $p$ ChiIV3). HSE: heat-shock element, MYB1AT: transcription factor MYB binding site, CGTCA-motif: methyl jasmonate (MeJA)-responsive element, ERELEE4: ethylene-responsive element, W-box: transcription factor WRKY binding site. B, Accumulation of ChilV3 transcripts in pepper leaves inoculated with $R$. solanacearum. Total RNA was extracted from leaves of 2-week-old pepper seedlings at different intervals of time postinoculation. $\mathbf{C}$ through $\mathbf{G}$, Transcript level analysis of ChiIV3 after treatments with phytohormones, including MeJA, abscisic acid (ABA), ethephon (ETH), brassinolide (BR), and salicylic acid (SA). Total RNA was extracted from 2-week-old pepper seedlings at different timepoints after treatments. For B through G, the expression level of ChiIV3 in untreated plants was set to 1. Error bars indicate standard deviation of three individual replicates. Asterisks indicate significant differences determined by Fisher's protected least significant differences test (one asterisk [*] indicates $P<0.05$, two $[* *] P<0.01)$. 
W4-box of the ChiIV3 promoter was critical for RSI, a 22-bp oligonucleotide containing a W4-box was used as a bait. A total of 43 positive clones were obtained and 11 clones were successfully amplified by PCR for further sequencing. Sequencing results hit three pepper genes with duplicates, including three NAD-dependent glyceraldehyde 3-P dehydrogenase (NADP, XP_006347401.1), two CBS domain-containing protein (CBS, XP_006344361.1), and six WRKY40 (NM_001325081). The bait and prey plasmids extracted from positive yeast clones were cotransformed into yeast strain Y1HGold to confirm binding activity of the three genes (Fig. 6A). NADP and CBS could not demonstrate the growth ability on SD/-Leu medium and were not further investigated (Fig. 6A). The positive result of WRKY40 was exciting and validated our previous finding that it also acts as a positive regulator in pepper resistance to $R$. solanacearum (Dang et al. 2013).

The chromatin immunoprecipitation (ChIP) procedure was performed to verify the binding of WRKY40 to the W4-box in ChiIV3 promoter. The oligonucleotides of the W4-box linked to the core CaMV35 promoter ( -46 to $+8 \mathrm{bp}$ ) was synthesized and fused to GUS reporter gene to create the reporter construct W4p35Score:GUS (Fig. 6B). Similarly, W4m-p35Score:GUS was also constructed with a 4-bp replacement of -TGAC- by -CCTT-, resulting in a sequence mutation in the W4-box (Fig. 6B). GV3101 cells carrying W4-p35Score:GUS or W4mp35Score:GUS were individually combined with p35S: WRKY40-HA (hemagglutinin) for chromatin isolation used for both ChIP and GUS activity measurements. ChIP-PCR results
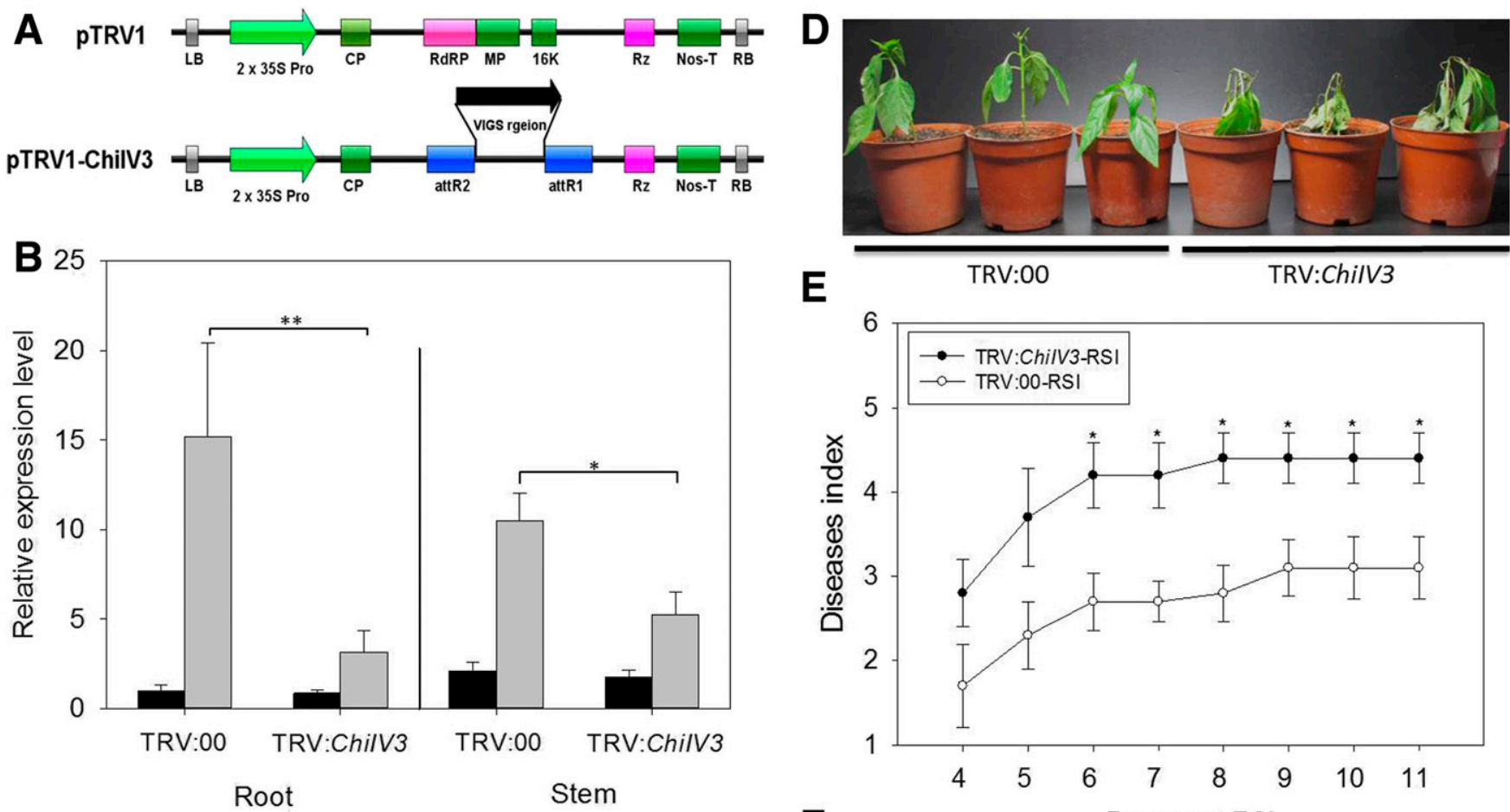

$\mathbf{E}$

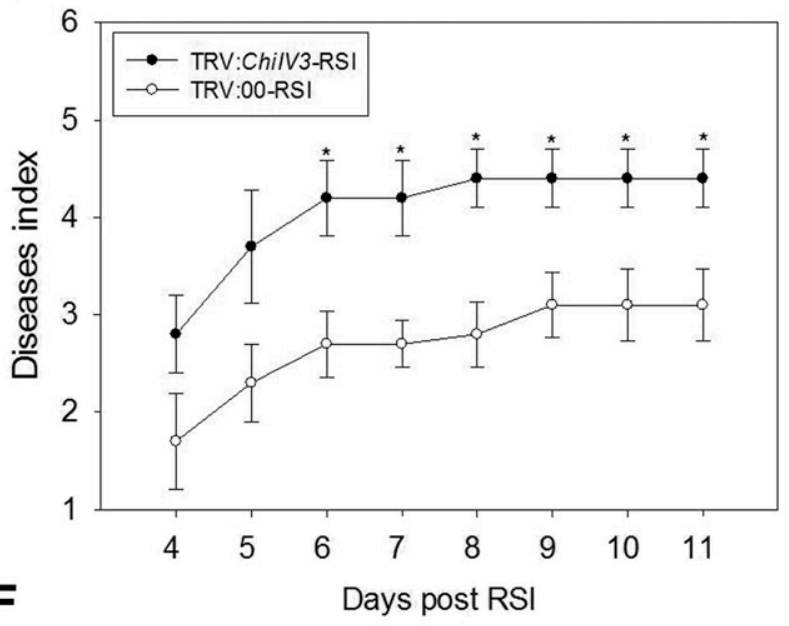

C Trypan blue

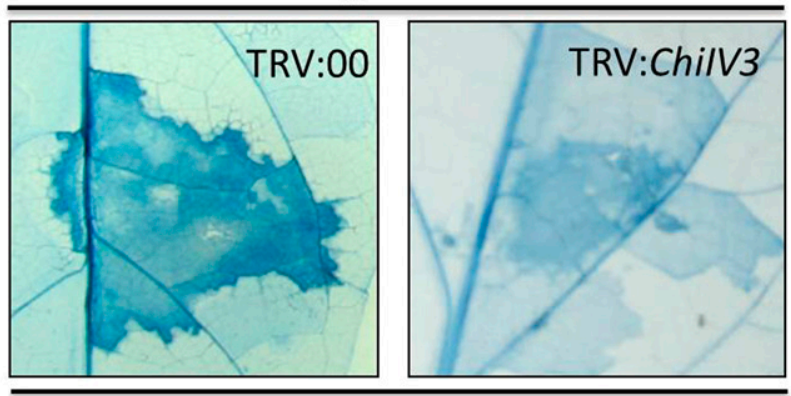

R. solanacearum
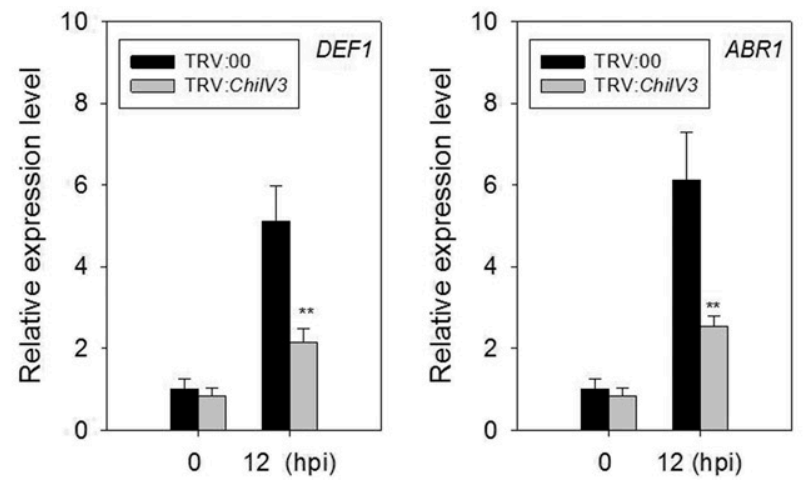

Fig. 2. Increased susceptibility of ChiIV3-silenced pepper plants to Ralstonia solanacearum inoculation. A, Lineal structure of ChiIV3-silenced vector. LB, left border; RB, right border. B, Silencing efficiency detection in roots and stems of ChiIV3-silenced pepper plants infected by R. solanacearum. Pepper plants with white leaves were used as a positive control. The transcript level of ChiIV3 in unsilenced pepper plants without treatment was set to 1 . C, Trypan blue staining was performed to evaluate cell death in ChiIV3-silenced pepper leaves infected by $R$. solanacearum. D, Disease symptoms of ChiIV3-silenced and unsilenced pepper plants inoculated with $R$. solanacearum. E, $R$. solanacearum-inoculated ChilV3-silenced and unsilenced pepper leaves were scored daily, using a disease index ranging from 0 to $4: 0$ (no wilting), 1 (1 to $25 \%$ wilted), 2 (26 to $50 \%$ wilted), 3 (51 to $75 \%$ wilted), and 4 (76 to $100 \%$ wilted or dead). F, Quantitative reverse transcription PCR was performed to investigate the transcript accumulation of defense-associated marker genes, including jasmonic acid-dependent $D E F 1$ and abscisic acid-responsive ABR1, in ChiIV3-silenced and unsilenced pepper leaves $12 \mathrm{~h}$ after $R$. solanacearum inoculation. The transcript level of marker genes in unsilenced pepper plants without treatment was set to 1 . In B, E, and F, error bars indicate standard deviation of three individual replicates. Asterisks indicate significant differences determined by Fisher's protected least significant differences test (one asterisk [*] indicates $P<0.05$, two $[* *] P<0.01)$. 
showed that WRKY40 product could directly bind to the W4box element of $p$ ChiIV3 (Fig. 6C). In addition, GUS activities driven by the W4-p35S core promoter were induced to high levels by transient expression of WRKY4O. However, the transient expression of WRKY4O did not induce an increased GUS activity driven by the W4m-p35S core (Fig. 6D). In RSI, the binding of WRKY40 to $p$ ChilV3 was significantly achieved when compared with the uninoculated control (Fig. 6E). These results verified that the W4-box was the key cis element required for WRKY4O to activate ChiIV3.

To further confirm the binding of WRKY40 to the W4-box in the ChiIV3 promoter, microscale thermophoresis (MST) experiments were performed using WRKY40-GFP fusion protein that is transiently expressed in pepper leaves. WRKY40-GFP labeled with NT-647-NHS fluorescent dye and synthetic W4box elements were subjected to thermophoresis experiments. Thermophoresis signal was plotted against ligand concentration to obtain a dose-response curve that facilitated precise evaluation of the binding affinity (Fig. 6F). The resultant binding curves showed that the wild-type W4-box was clearly associated with WRKY40 protein and binding intensity increased with the increase in concentration of target DNA.

WRKY40 modulates ChiIV3 promoter activity in pepper.

To examine whether ChiIV3 is regulated by WRKY40, fulllength cDNAs of WRKY4O were fused to HA-tag driven by
CaMV35 promoter to generate p35S:WRKY40-HA (Fig. 7A). GV3101 cells containing $p 35 S: W R K Y 40-H A$ were infiltrated into the leaves of 8-week-old pepper. The protein expression level of WRKY40-HA was confirmed by immunoblot analysis at 48 hpi (Fig. 7B). Additionally, the transcriptional level of ChiIV3 was found to be significantly induced by WRKY4O overexpression (Fig. 7C). Agrobacterium-mediated cotransformation of WRKY4O-HA and ChiIV3 promoter:GUS revealed that WRKY4O significantly activated GUS expression driven by the ChiIV3 promoter (Fig. 7D). GUS activity was also determined on WRKY4O knockdown pepper plants. In comparison with that in unsilenced plants, ChiIV3 promoter-driven GUS activities were found to be significantly reduced in WRKY40-silenced pepper plants (Fig. 7E). Altogether, these results indicated that transcription of $p C h i I V 3$ is mediated by WRKY40.

\section{DISCUSSION}

Plant PR proteins induced by pathogen infection play vital roles in disease resistance (Hadwiger 2015; Li et al. 2011; Murray et al. 2005; Vasavirama and Kirti 2012; Zhang and Mou 2009). Plant chitinases are comprised of a family of universal PR proteins that can catalyze the hydrolysis of fungal chitin to generate chitooligosaccharides. Released chitin fragments are found to be involved in PTI during fungal pathogen infection
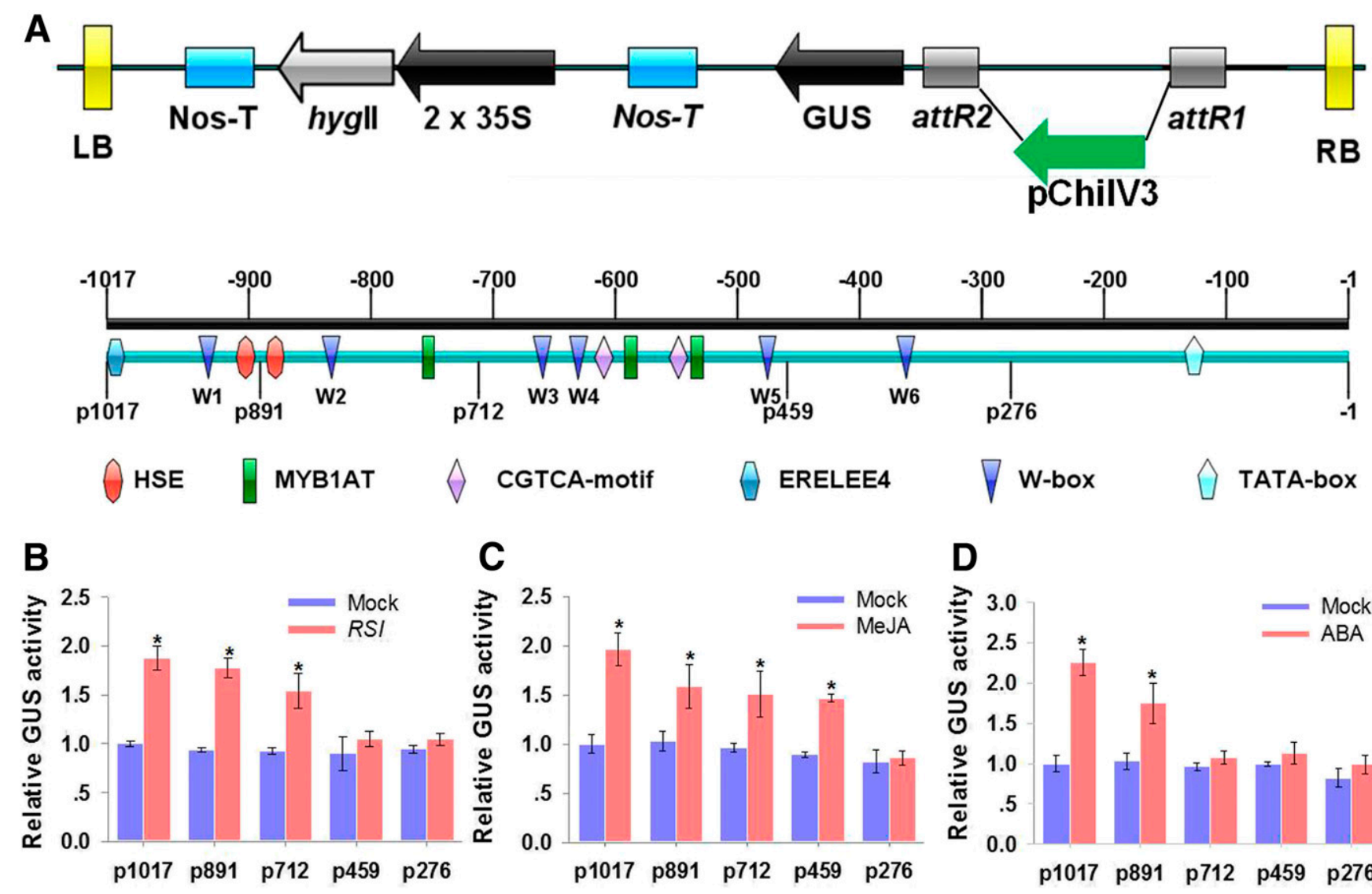

CGTCA-motif

ERELEE4

W-box

TATA-box
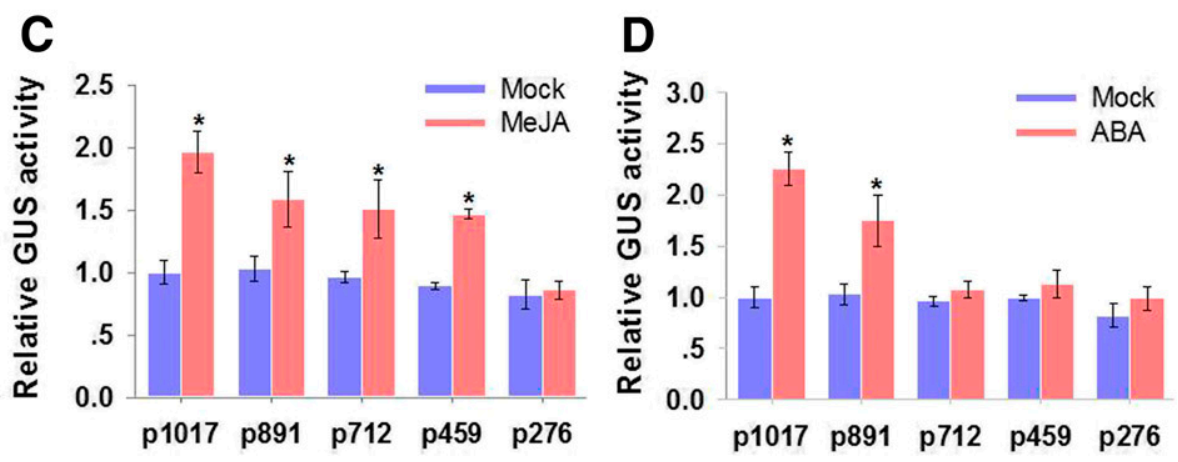

Fig. 3. ChilV3 promoter activation in response to Ralstonia solanacearum infection and hormone treatments in tobacco plants. A, Schematic diagram of the vector of ChiIV3 promoter ( $p$ ChiIV3), its deletions (p1017, p891, p712, p459, and p276) and cis-element distribution in five deletions of $p$ ChiIV3. LB, left border; RB, right border; W1-W6 represent W-boxes found in ChiIV3 promoter. B to D, ChilV3 promoter activation in response to R. solanacearum inoculation and exogenous methyl jasmonate (MeJA) and abscisic acid (ABA) in leaves of transgenic tobacco plants harboring 5'-ChiIV3-GUS chimeric constructs. The pathogen and exogenous MeJA- and ABA-treated transgenic tobacco leaves were harvested $24 \mathrm{~h}$ posttreatment for analyzing $\beta$-glucuronidase (GUS) activities. The GUS activities in transgenic tobacco leaves harboring a deletion of p1017 (full-length promoter) treated with mock solution $\left(\mathrm{MgCl}_{2}\right.$ or hormone solution) were set to 1. Error bars indicate standard deviation of three individual replicates. Asterisks indicate significant differences determined by Fisher's protected least significant differences test (one asterisk $[*]$ indicates $P<0.05$, two $[* *] P<0.01$ ). 
(Chen et al. 2018). However, the transcription of plant chitinase genes is not strictly controlled by fungal pathogens, because certain bacteria, viruses, and oomycetes without chitin in their cell walls have been found to induce chitinase gene expression (Guan and Chye 2008; Hong and Hwang 2002; Hong et al. 2000; Mishra et al. 2012). This suggested that plant chitinases exert their roles in resistance to several kinds of pathogens, including bacteria. Recently, a pepper class IV chitinase showed that CaChitIV interacted with CaPIK1 and promoted CaPIK1-triggered cell death against Xanthomonas campestris pv. vesicatoria infection (Kim et al. 2015). Our previous study reported that ChiIV3 showed antifungal activity against $P$ hytophthora capsici and a W5-box element in the promoter was involved in the response to $P$. capsici infection (Liu et al. 2017). This was consistent with the conclusion that chitinase can act as an antifungal protein and exhibit critical roles in response to fungal invasion (Maximova et al. 2006; Kashyap and Deswal 2017; Khan et al. 2017; Kim et al. 2015; Miyamoto et al. 2012; Richa et al. 2016; Tobias et al. 2007; Yang et al. 2014). In this study, we uncovered the role of ChiIV3 during $R$. solanacearum infection, which causes severe bacterial wilt disease in pepper plant. ChilV3-silencing pepper plants showed high susceptibility to $R$. solanacearum, coupled by severe disease symptoms and low expression level of defense-associated marker genes. Subsequently, pepper ChilV3 acted as a plant PR protein required for resistance against $R$. solanacearum.
The pepper ChilV3 gene was expressed in a multiple regulatory mode, especially when induced by $R$. solanacearum infection. The expression of chitinase genes have been highly regulated to coordinate with plant growth and development as well as the response to biotic and abiotic stress conditions (Filippi et al. 2016; Karmakar et al. 2016; Kim et al. 2014a; Mészaros et al. 2014; Wu et al. 2017). Transcriptional expression of a gene is commonly coordinated by transcription factors, which bind and recognize the promoter composed of multiple cis-regulatory elements (Kim et al. 1997; Zhao et al. 2007, 2012). The present study discovered that the expression of ChilV3 was significantly induced by $R$. solanacearum, MeJA, and ABA, which led us to study the cis elements present in the ChiIV3 promoter. Using PlantCARE software, six Wboxes, three MYB1ATs, two HSEs, three MYB1ATs, and two CGTCA-motifs were predicted from the ChiIV3 promoter. Through promoter GUS fusion experiments, the W4-box element was found to be critical in response to $R$. solanacearum infection. In addition, the W6-box located from -339 to $-334 \mathrm{bp}$ and the MYB1AT element located from -735 to $-730 \mathrm{bp}$ were found to be involved in the response to exogenous MeJA and ABA, respectively. Even though the function of cis elements in the ChiIV3 promoter had not been completely elucidated, current findings suggested that the ChiIV3 gene was controlled by complex regulatory modes. The phytohormones anchored various cis elements to modulate ChiIV3 expression.

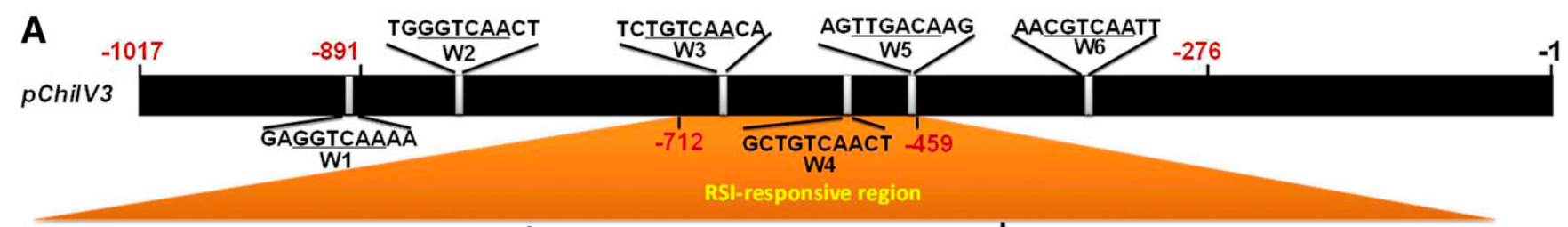

B
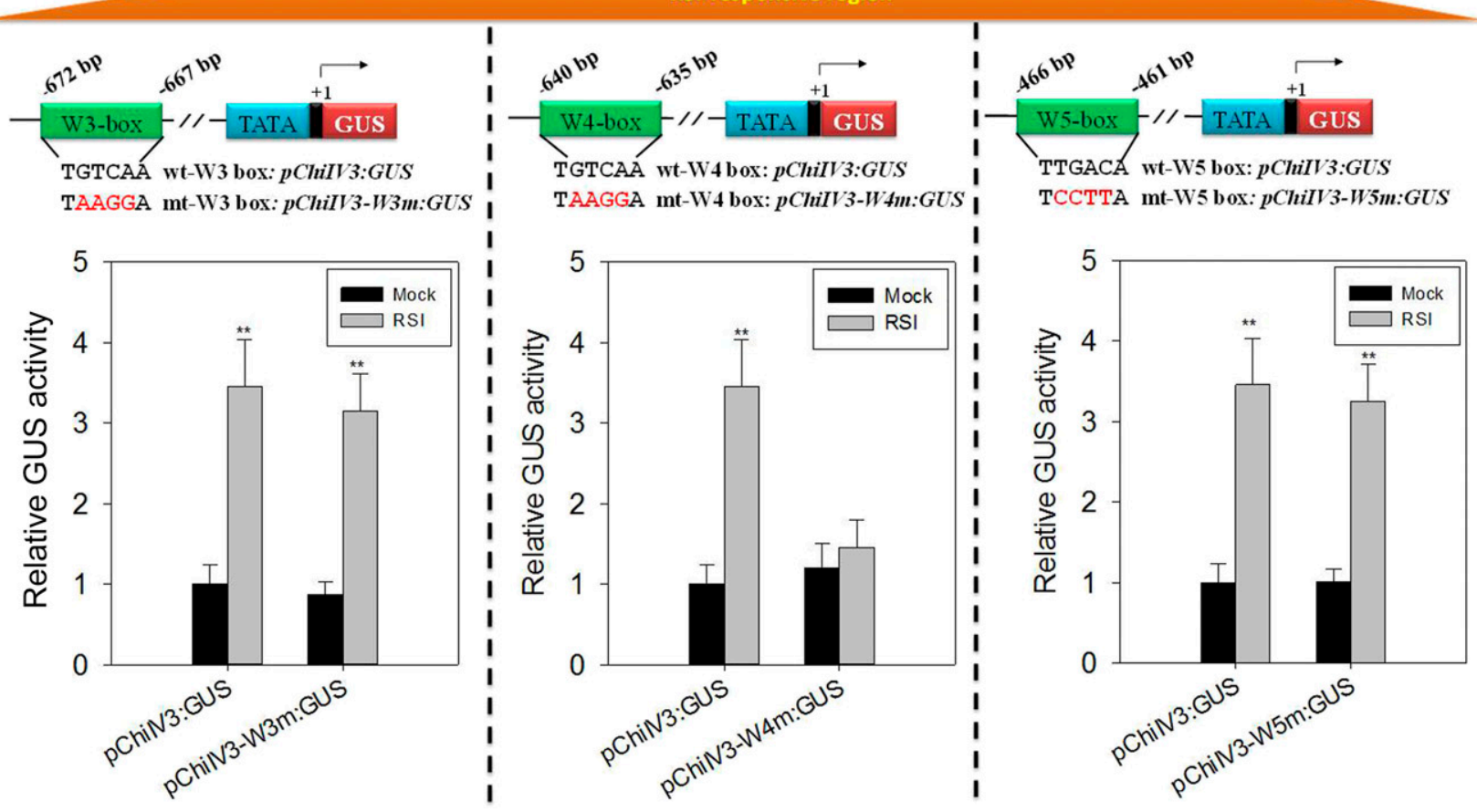

Fig. 4. Site-directed mutagenesis analysis of ChilV3 promoter in response to Ralstonia solanacearum infection in pepper plants. A, Locations of W-boxes in ChiIV3 promoter ( $p$ ChiIV3). Numbers above the bar indicate the position of five $p$ ChiIV3 deletions. The underlined nucleotides represent W-boxes. B, The schematic diagram of $p$ ChiIV3:GUS and its W-box-mutant constructs ( $p C h i I V 3-W 3 m: G U S, p C h i I V 3-W 4 m: G U S$, or $p C h i I V 3-W 5 m: G U S)$ and their $\beta$-glucuronidase (GUS) activities initiated by $R$. solanacearum inoculation in pepper leaves. The mutated nucleotides of the W-box are shaded. The GV3101 cells containing $p$ ChiIV3:GUS or its W-box mutant constructs were penetrated into the pepper leaves and were maintained in the greenhouse for $24 \mathrm{~h}$, followed by $R$. solanacearum inoculation. The pathogen-inoculated leaf tissues were harvested for GUS activity measurements at $48 \mathrm{~h}$ postinoculation. Values are means \pm standard deviation (SD) $(n=6)$. Error bars indicate SD. Asterisks indicate significant differences determined by Fisher's protected least significant differences test (one asterisk [*] indicates $P<0.05$, two [**] $P<0.01$ ). 
Additionally, the W5-box was involved in response to $P$. capsici infection (Liu et al. 2017), while the W4-box was essential for $R$. solanacearum induction.

WRKY proteins depend on the conserved WRKY domains to bind to W-box cis elements in the promoters of their targets (Agarwal et al. 2011; Eulgem 2006; Eulgem et al. 1999, 2000; Rushton et al. 2010, 2012). The specificity of targets is determined by WRKY domains of WRKY proteins and W-boxes in target gene promoters. Previous studies have reported that nucleotide sequences flanking W-box elements demonstrated a certain degree of binding specificity to WRKY proteins (Brand et al. 2013; Ciolkowski et al. 2008; Gao et al. 2016). The specificity might also be modulated through posttranslational modification of WRKY proteins. Various enzymes, such as MAPKs and MAPK kinases (Adachi et al. 2016; Giri et al. 2013; Ishihama et al. 2011, 2014; Kim and Zhang 2004; Liu et al. 2004; Miao et al. 2007; Pecher et al. 2014; Shen et al. 2012; Weyhe et al. 2014b; Yoo et al. 2014; Zhang et al. 2012), $\mathrm{Ca}^{2+}$-dependent protein kinases (Gao and He 2013), VQ-motifcontaining proteins (Cheng et al. 2012; Lei et al. 2017; Pecher et al. 2014; Wang et al. 2015; Weyhe et al. 2014a), calmodulin (Park et al. 2005), histone deacetylases (Kim et al. 2008; Singh et al. 2014a and b), and E3 ubiquitin ligase (Miao and Zentgraf 2010), have been found to interact with WRKY proteins. Some WRKY proteins can even interact with each other in plants (Chen et al. 2010). The interaction of WRKY with other proteins can often lead to the change in transcriptional activation activity or targeting specificity. Through yeast one-hybrid, ChIP, and MST assays, the W4-box was confirmed to be the critical element targeted by WRKY40. Moreover, the W4-box seemed to be a unique binding site for WRKY40, because sitedirected mutagenesis in the W4-box completely impaired the induction of ChiIV3 by WRKY40. It is still unclear whether the specificity of ChilV3 targeted by WRKY40 is determined through protein-protein interaction. The identification of interacting pepper proteins of WRKY40 would provide new insight into WRKY40-ChiIV3 mediated pepper resistance to $R$. solanacearum infection.

Arabidopsis WRKY40 together with WRKY18 and WRKY60 are three paralogous WRKY transcription factors that regulate
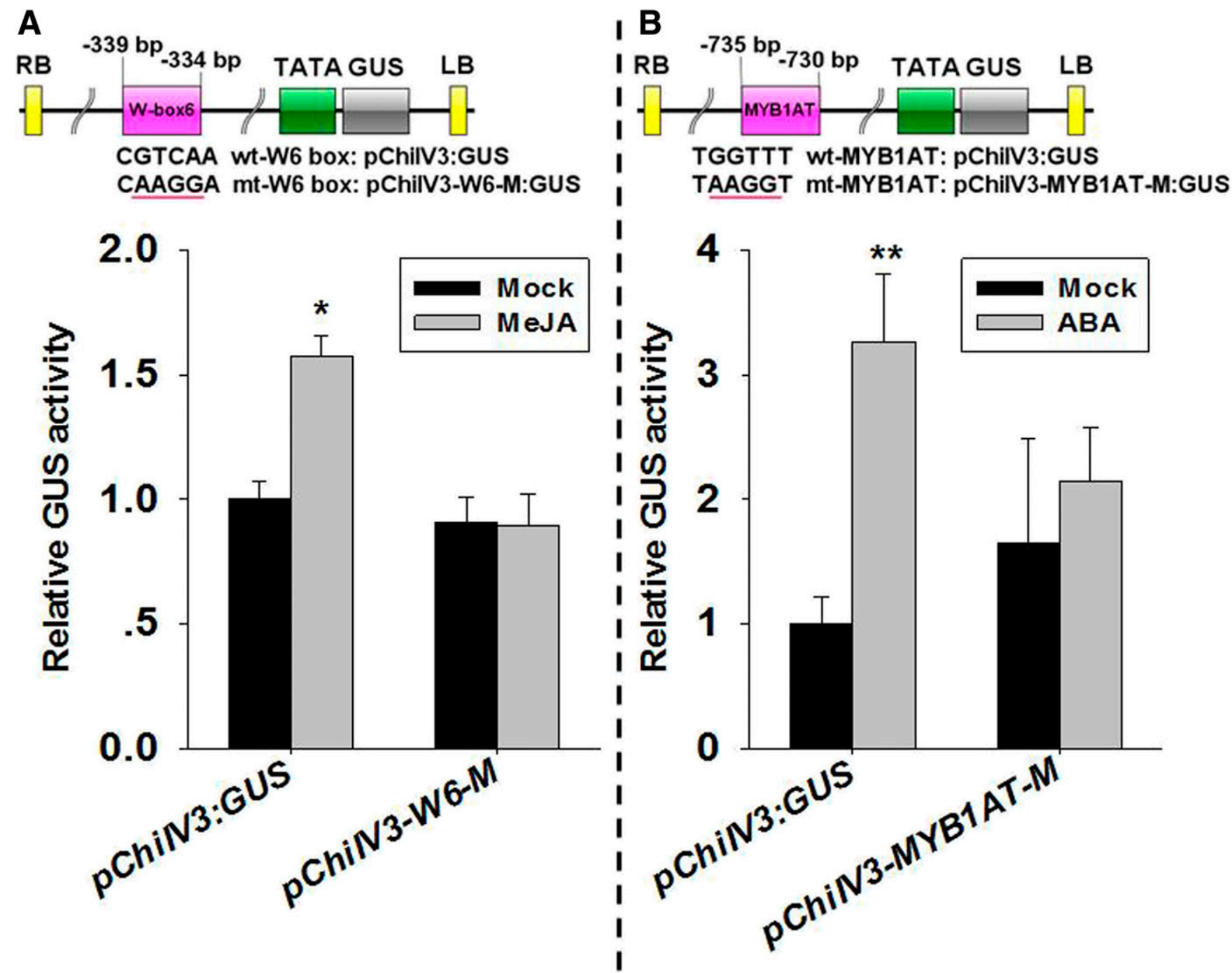

Fig. 5. Site-directed mutagenesis analysis to confirm the cis elements of $p$ ChiIV3 in response to exogenous methyl jasmonate (MeJA) and abscisic acid (ABA) treatments in pepper plants. A and B, The schematic representation of serial mutant constructs of $p C h i I V 3: G U S$ ( $p C h i I V 3-W 4-M: G U S, p C h i I V 3-W 6-M$ :GUS, and $p C h i I V 3-M Y B 1 A T-M: G U S$ ) and the measurement of $\beta$-glucuronidase (GUS) activity in response to $R$. solanacearum inoculation and exogenous MeJA and ABA treatments, respectively. The mutated nucleotides are underlined. At $24 \mathrm{~h}$ postinoculation or treatment, the infiltrated pepper leaves were harvested for measuring GUS activity. Error bars indicate standard deviation of six individual replicates. Asterisks indicate significant differences determined by Fisher's protected least significant differences test (one asterisk [*] indicates $P<0.05$, two [**] $P<0.01$ ). 
plant defense in a complex pattern (Liu et al. 2012). Three WRKY proteins can form both homo-and hetero-complexes and exhibit physical interaction with themselves or with each other through a basic leucine zipper motif at their N-terminus (Xu et al. 2006). The transcription of WRKY40 was suppressed by $\mathrm{ABA}$ and negatively regulates $\mathrm{ABA}$-dependent signaling by direct repression of ABA-responsive $A B I 4$ and $A B I 5$ genes (Liu et al. 2012). With transient expression in Arabidopsis thaliana protoplasts, WRKY40 localizes to phytochrome Bcontaining nuclear bodies (PNBs). However, WRKY40 can relocalize from PNBs to nucleoplasm in the presence of ABA in a dynamic and phosphorylation-dependent manner (Geilen and Böhmer 2015). WRKY40 transcription in pepper was activated by WRKY 6 before regulating the $R$. solanacearum resistance as well as tolerance to high temperature and high humidity. Numerous targets of WRKY40 have been identified in our previous study, including $\mathrm{CaC} 3 \mathrm{H} 14$ encoding a tandem $\mathrm{CCCH}$ zinc finger protein and $C a C D P K 15$ encoding a calciumdependent protein kinase (Shen et al. 2016a; Qiu et al. 2018). In this study, we reported that ChilV3 was a novel target of WRKY40 involved in resistance to $R$. solanacearum infection. It can be concluded that WRKY40 in pepper could directly modulate several targets to execute its regulatory function. However, target genes involved in tolerance to high temperature or high humidity have not yet been experimentally proven.

Besides RSI and phytohormones, other unknown factors must be involved in ChilV3 transcription regulation because its promoter contained multistress-responsive $c$ is elements. In addition, roles of the W1-box, W2-box, and W3-box have not been explained. Considering that the W-box is the binding target of WRKY protein, other WRKY transcription factors, including WRKY40, might have jointly regulated ChiIV3 expression. Moreover, transcription of ChiIV3 was reduced by the application of SA, ETH, or BR. Negative regulation of these three phytohormones possibly occurred through the cis elements in the ChiIV3 promoter. It will be interesting to determine which cis elements were involved in negative regulation of ChilV3 transcription in future studies.

Based on the comprehensive evidence in the present study, a proposed model was constructed to explain the transcription of ChiIV3 modulated by WRKY40 (Fig. 8). When pepper plants are infected by $R$. solanacearum, signal is transmitted to the nuclei, thus promoting the transcription of WRKY4O (Dang et al. 2013, 2014). WRKY40 protein directly binds to promoters of ChiIV3 in a W-box-dependent manner. The enhanced expression of ChiIV3 then induces defense response and expression of defense-associated marker genes, such as $A B R 1$ and $D E F 1$. The pepper resistance to $R$. solanacearum was successfully elucidated through this regulatory mode.

In conclusion, the present study reported that pepper chitinase ChiIV3 played an important role in resistance to bacterial wilt. The transcription of the ChilV3 gene was induced by $R$. solanacearum, JA, and ABA through cis-regulatory elements in the promoter region. The W4-box was found to be essential
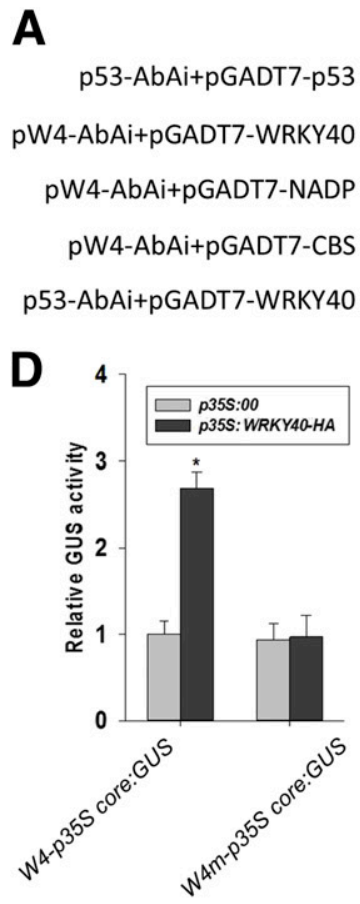

$\mathrm{SD} /-\mathrm{Leu}+300 \mathrm{ng} / \mathrm{mL} \mathrm{AbAr}^{\mathrm{r}}$
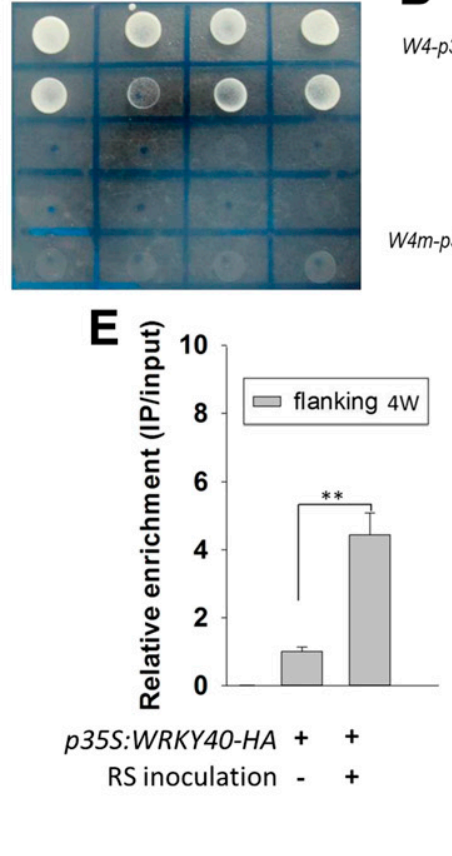
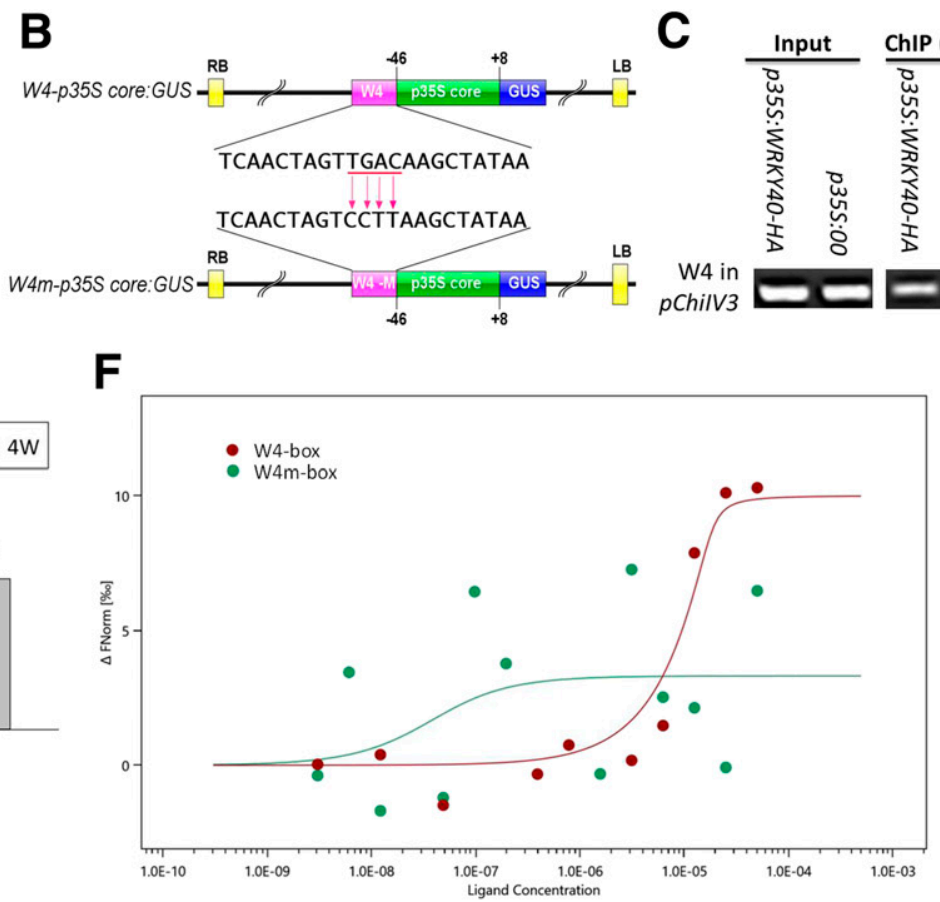

Fig. 6. WRKY40 acts as a positive regulator of ChiIV3. A, Yeast one-hybrid analyses of the upstream regulators of ChiIV3. Bait plasmids coding WRKY40, $N A D P$, and $C B S$ were retransformed into yeast containing bait plasmids and were planted on SD/-Leu medium supplemented with 300 ng of Aureobasidin A $\left(\mathrm{AbA}^{\mathrm{r}}\right)$ per milliliter. B, Schematic diagram of the W4-p35Score:GUS vector and W4m-p35Score:GUS. M, mutant; LB, left border; RB, right border. Mutated nucleotides are underlined. C, WRKY40 directly binds to W4-containing fragment of $p$ ChiIV3 in nonstressed pepper plants analyzed by chromatin immunoprecipitation (ChIP) assay. Pepper leaves expressing WRKY40-HA (hemagglutinin) were harvested at $48 \mathrm{~h}$ postinoculation for the isolation of chromatins and the ChIP assay. D, The transient overexpression of WRKY40 triggered the expression of $\beta$-glucuronidase (GUS) driven by W4-p35S core in a W4 dependent manner. GUS activity in pepper leaves coinfiltrated with GV3101 cells containing pW4-p35Score:GUS and p35S:00 was set to 1 . E, R. solanacearum inoculation improved the binding of WRKY40 to W4-box containing $p C h i I V 3$. F, An MST-based binding experiment was performed to confirm the binding of purified WRKY40 with W4-box and W4-box mutation. The binding states of the molecule display different thermophoretic depletion profiles. The difference in thermophoresis can be plotted as a change in normalized fluorescence versus ligand concentration to obtain a binding curve. In $\mathrm{D}$ and $\mathrm{E}$, data represent mean \pm standard deviation (SD) of three independent experiments. Error bars indicate SD. Asterisks indicate significant differences determined by student's $t$ test (one asterisk [*] indicates $P<0.05$, two [**] $P<0.01)$. 
for $R$. solanacearum induction and it was the binding site of WRKY40. The collective data supported that ChiIV3 was a novel resistance gene directly regulated by WRKY40 to stimulate plant resistance against $R$. solanacearum infection. These results provide insight into the function and regulation of pepper ChiIV3 during RSI.

\section{MATERIALS AND METHODS}

Plant materials and cultivation.

Seeds of pepper inbred line yanshan01 (Capsicum annuum cv. yanshan01) and two tobacco cultivars (Nicotiana tabacum K326 and $N$. benthamiana) were collected by the pepper breeding group in Fujian Agriculture and Forestry University (Fujian Province, China). Pepper and tobacco seeds were germinated in a steam-sterilized soil mix (peat moss: per liter [2:1, $\mathrm{vol} / \mathrm{vol}]$ ) in plastic pots. Following germination for 2 weeks, the seedlings were transferred to larger pots and were maintained in a growth room at $25^{\circ} \mathrm{C}, 60$ to $70 \mathrm{mmol}$ photons $\mathrm{m}^{-2} \mathrm{~s}^{-1}, 70 \%$ relative humidity, and a 16-h light and 8-h dark photoperiod.

\section{Pathogen inoculation and exogenous application of MeJA and ABA.}

Ralstonia solanacearum was isolated and stored in the pepper breeding center of Fujian Agriculture and Forestry University. For preparation of $R$. solanacearum, stem exudates and stem vascular parts from pepper plants were purified using the tetrazolium chloride method (van Elsas et al. 2001). The strains were incubated overnight at $200 \mathrm{rpm}, 28^{\circ} \mathrm{C}$ in SPA medium
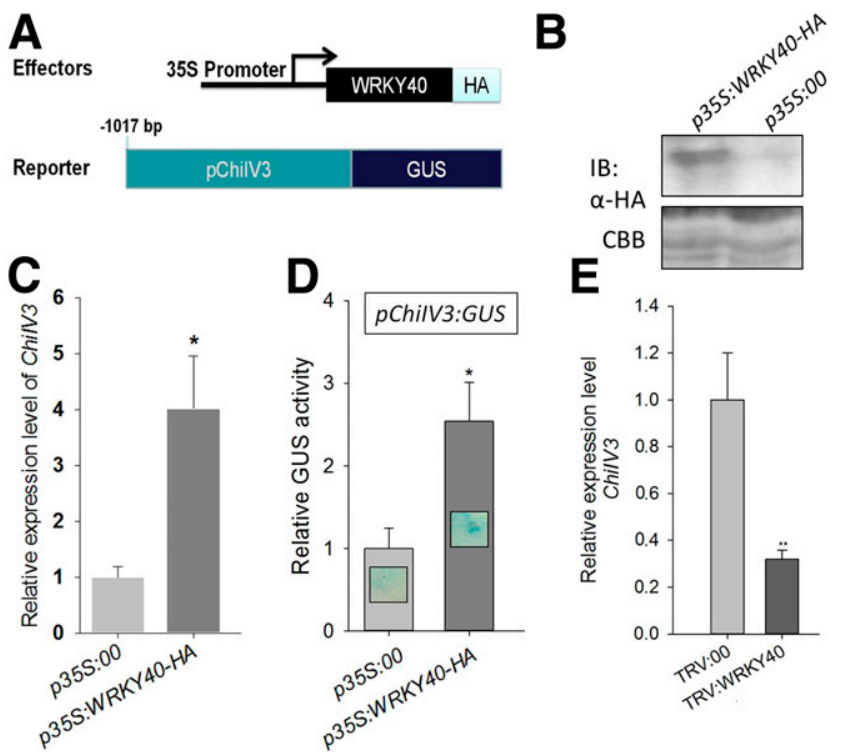

Fig. 7. WRKY40 triggers the expression of $\beta$-glucuronidase (GUS) driven by $p$ ChiIV3. A, The schematic diagram of effector and reporter vectors. B, Immunoblot analysis of WRKY40-HA (hemagglutinin) transiently expressed in pepper leaves at $48 \mathrm{~h}$ postinoculation (an anti-HA antibody was used to detect WRKY40-HA proteins on immunoblot). IB, immunoblotting. C, Overexpression of WRKY4O induced the transcript expression of ChilV3, analyzed by quantitative reverse transcription PCR. The transcript level of ChilV3 in pepper leaves infiltrated with GV3101 cells containing p35S:00 (empty vector) was set to 1 . D, The expression of GUS driven by $p C h i I V 3$ was improved by transient overexpression of WRKY40. GUS activity of pepper leaves coinfiltrated with $p C h i l V 3: G U S$ and $p 35 S: 00$ was set to 1 . E, WRKY40-silencing partially stops the transcript accumulation of ChiIV3 in healthy pepper plants. The transcript level of ChiIV3 in unsilenced pepper plants was set to 1 . In $\mathrm{C}$ to $\mathrm{E}$, asterisks indicate significant differences determined by Fisher's protected least significant differences test (one asterisk [*] indicates $P<0.05$, two [**] $P<0.01$ ).
( $200 \mathrm{~g}$ of potato, $20 \mathrm{~g}$ of sucrose, $3 \mathrm{~g}$ of beef extract, and $5 \mathrm{~g}$ of tryptone in 1 liter of double-distilled $[\mathrm{dd}] \mathrm{H}_{2} \mathrm{O}$ ). The cell pellet was collected using low-speed centrifugation and was suspended in sterile $10 \mathrm{mM} \mathrm{MgCl}_{2}$. Suspension was then adjusted to an optical density at $595\left(\mathrm{OD}_{595}\right)$ of $0.8\left(10^{8} \mathrm{CFU} / \mathrm{ml}\right)$. Ten microliters of $R$. solanacearum suspension was injected into the roots of transgenic tobacco or ChiIV3-silenced pepper leaves using a syringe and, later, the leaves were harvested at different time intervals for further assay. For exogenous application of phytohormones, plants were sprayed with $100 \mu \mathrm{M}$ ABA and $100 \mu \mathrm{M}$ MeJA dissolved in $\mathrm{ddH}_{2} \mathrm{O}$. Plants sprayed with $\mathrm{ddH}_{2} \mathrm{O}$ were kept as a control.

\section{Amplification of 5' deletions or site-directed mutagenesis of pChilV3 by PCR.}

pChiIV3 and its $5^{\prime}$ deletion derivatives $(-1,017,-891,-712$, -459 , and 276 to +1 ; the first nucleotide of the initiation codon ATG was set as +1) were constructed using Gateway technology (Liu et al. 2017). Site-directed mutagenesis of $p C h i I V 3$ was performed using the primers $p$ ChiIV3-W3m-F, $p$ ChiIV3-W4m-F, pChiIV3-W5m-F, pChiIV3-W6m-F, and pChiIV3-MYB1AT-F (Supplementary Table $\mathrm{S} 1$ ). The attB-containing $\mathrm{PCR}$ product of $p$ ChiIV3 and its $5^{\prime}$ deletion derivatives were cloned into the satellite vector $\mathrm{pDONR} 207$ via BP reaction. After confirmation by sequencing, the generated BP products were transferred into the destination vector $\mathrm{pMDC} 163$ via $\mathrm{LR}$ reaction to generate pChiIV3, pChilV3-W3-M, pChilV3-W4-M, pChiIV3-W5-M, pChilV3-W6-M, and $p$ ChilV3-MYB1AT-M, respectively.

\section{VIGS of ChiIV3 in pepper plants.}

Loss-of-function of ChilV3 in pepper plant in response to $R$. solanacearum inoculation was performed using VIGS

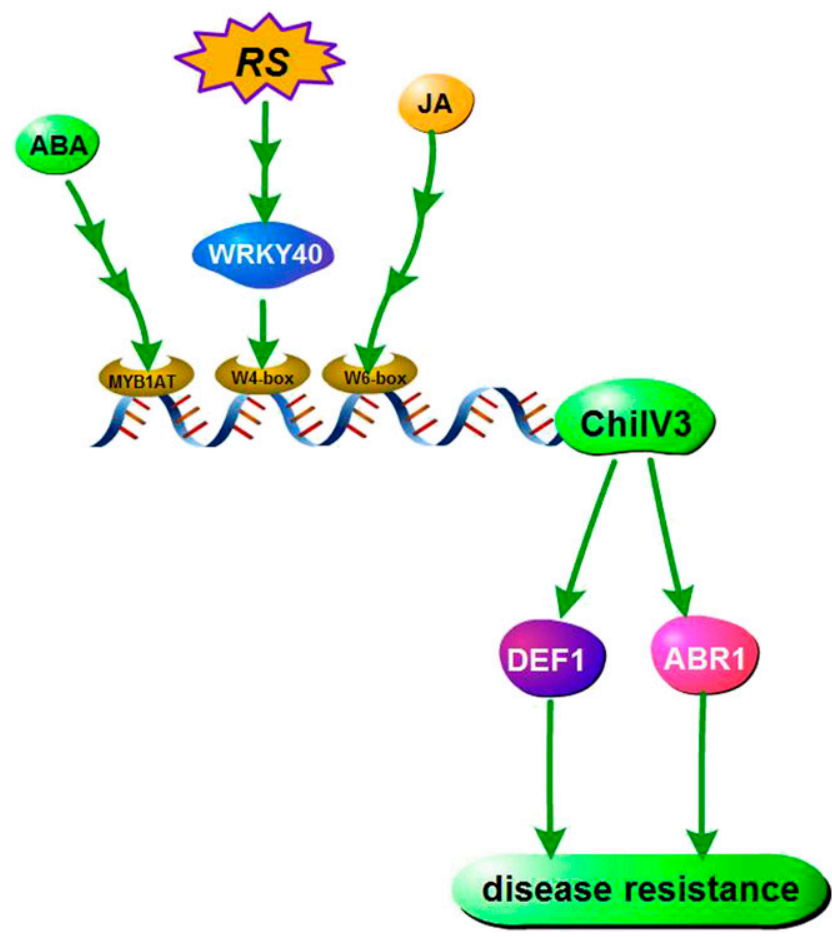

Fig. 8. Proposed model for the role of ChiIV 3 modulated by WRKY 40 in defense signaling. The pepper WRKY transcription factor WRKY40 regulates expression of ChilV3 via the W-box in the upstream promoter. ChiIV3 products trigger defense response and the expression of defense-associated marker genes including $D E F 1$ and $A B R 1$ against $R$. solanacearum. RS, Ralstonia solanacearum. Arrows indicate positive regulation. 
technology as described previously (Liu et al. 2016). Briefly, a specific $3^{\prime}$ untranslated region fragment of ChilV3 that was approximately $200 \mathrm{bp}$ in length was employed to construct the VIGS vector, the specificity of the region was confirmed by BLASTN against pepper genome databases (Pepper Genome Platform). The fragment was amplified and cloned into the VIGS vector pTRV2, using Gateway technology to yield pTRV2ChiIV3. After confirmation by sequencing, pTRV2-ChiIV3 with the pTRV1 vector or pTRV2 vector were separately transformed into GV3101 cells. A 5-ml culture of each strain was grown overnight in YEP medium (per milliliter, $10 \mathrm{mg}$ of yeast extract, $10 \mathrm{mg}$ of peptone, and $5 \mathrm{mg}$ of $\mathrm{NaCl}$ ) supplemented with appropriate antibiotics (per milliliter, $75 \mathrm{mg}$ of kanamycin and $75 \mathrm{mg}$ of rifampicin). GV3101 cells were harvested by centrifugation and were resuspended in infiltration buffer $(10 \mathrm{mM}$ $\mathrm{MgCl}_{2}, 10 \mathrm{mM}$ morpholineethane sulfonic acid [MES], $\mathrm{pH}$ 5.7, and $200 \mu \mathrm{M}$ acetosyringone) and adjusted to $\mathrm{OD}_{600}=0.5$. GV3101 strains containing the pTRV1 vector and pTRV2 or pTRV2-ChiIV3 were mixed at a 1:1 ratio, were shaken at room temperature for $3 \mathrm{~h}$, and were coinfiltrated into the cotyledons of pepper seedlings. The VIGS-treatment pepper plants were grown at $25^{\circ} \mathrm{C}$ with a 16 -h light and 8 -h dark photoperiod cycle.

\section{Fluorometric assays and histochemical staining for GUS activity.}

For GUS activity, total protein was extracted from stresstreated leaves in transgenic tobacco, ChilV3-silenced pepper, or pepper transiently expressing the GUS-tag vectors and was quantified according to the method described by Mou et al. (2013), with a slight modification. The rate of $p$-nitrophenol release $(\gamma=415 \mathrm{~nm})$ was measured using a microplate reader (Biotek) to confirm the GUS activity. For histochemical staining, infected pepper leaves were harvested and immersed in GUS staining solution ( $200 \mathrm{mM}$ phosphate buffer, $\mathrm{pH} 7.0$, containing $100 \mathrm{mM} \mathrm{K}_{3}\left[\mathrm{Fe}(\mathrm{CN})_{6}\right], 100 \mathrm{mM} \mathrm{K}_{4}\left[\mathrm{Fe}(\mathrm{CN})_{6}\right]$, 1,000 mM EDTA-Na ${ }_{2}$, and 0.1\% 5-bromo-4-chloro-3-indolyl$\beta$-D-glucuronide) and were incubated at $37^{\circ} \mathrm{C}$ overnight, then, cleaned with $70 \%$ ethanol $(\mathrm{EtOH})$.

\section{Agrobacterium-mediated transient expression assay.}

Agrobacterium-mediated transient expression assays were performed in pepper plants according to a previously described method, with a slight modification (Liu et al. 2015). Agrobacterium cells containing different constructs were grown in YEP medium (per liter, $10 \mathrm{~g}$ of yeast extract; $10 \mathrm{~g}$ of peptone; $5 \mathrm{~g}$ of sodium chloride; $\mathrm{pH} \mathrm{7.0)}$ overnight at $28^{\circ} \mathrm{C}$, and the cell pellet was collected, by centrifugation for $5 \mathrm{~min}$ at $8,000 \mathrm{rpm}$, and was suspended in the infiltration medium $(10 \mathrm{mM} \mathrm{MgCl}$, $10 \mathrm{mM}$ MES, pH 5.7, and $200 \mu \mathrm{M}$ acetosyringone). The adjusted cell suspension was penetrated into pepper leaves using a needleless syringe. The treated pepper plants were maintained at $25^{\circ} \mathrm{C}$ in greenhouse.

\section{Histochemical staining.}

3, 3'-Diaminobenzidine tetrahydrochloride (DAB) and trypan blue staining procedures were performed as described in our previous studies (Liu et al. 2015, 2016). For DAB staining, treated pepper leaves were harvested and immersed in DAB solution (1 $\mathrm{mg}$ of DAB per milliliter). At $8 \mathrm{~h}$ posttreatment, DAB-stained pepper leaves were boiled in absolute ethanol for destaining. For trypan blue staining, pepper leaves were harvested and boiled in trypan blue solution $(10 \mathrm{ml}$ lactic acid,

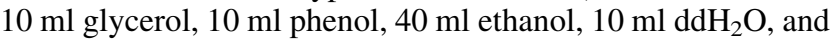
$1 \mathrm{ml}$ trypan blue) for $5 \mathrm{~min}$, were kept at room temperature for 8 $\mathrm{h}$, then, were transferred to chloral hydrate solution $(2.5 \mathrm{~g}$ of chloral hydrate per milliliter dissolved in distilled water) and were boiled for $25 \mathrm{~min}$, for destaining.

\section{Yeast one hybrid.}

Bait construct, pW4-AbAi containing a 22-bp pChiIV3 fragment with a W4-box (ATAAGCTGTCAACTAAGTGAC GAGCTAT) was transformed into yeast strain Y1HGold according to the protocol handbook (Clontech). To determine the expression profile of the bait construct in yeast, the transformed yeast strain was subjected to grow on SD/-Leu medium supplemented with gradient concentrations of Aureobasidin A $\left(\mathrm{AbA}^{\mathrm{r}}\right)(50,100,200,300$, and $400 \mathrm{ng} / \mathrm{ml})$. For the W4-box, a concentration of $300 \mathrm{ng} / \mathrm{ml}$ was enough to suppress the background activity of the bait yeast strain. Pepper cDNA library fused with pGADT7-Rec AD was created from pepper leaves infected by $R$. solanacearum and was screened using the bait yeast strain. The transformed yeast cells were plated on SD/-Leu medium supplemented with $300 \mathrm{ng}$ of $\mathrm{AbA}^{\mathrm{r}}$ per milliliter and were incubated at $30^{\circ} \mathrm{C}$ for 3 to 5 days. PCR was performed to detect the insertion of the prey vector and was subjected to sequence analysis. Y1HGold strains transformed with p53AbAi+pGADT7-p53 and p53-AbAi+pGADT7-WRKY40 were used as positive and negative controls, respectively.

\section{Western blotting and ChIP assay.}

Western blotting was performed following the method described in our previous study (Liu et al. 2017). Protein extraction buffer (10\% glycerol, $25 \mathrm{mM}$ Tris- $\mathrm{HCl}, \mathrm{pH} 7.5$, $150 \mathrm{mM} \mathrm{NaCl}, 1 \mathrm{mM}$ EDTA, 2\% Triton X-100, $10 \mathrm{mM}$ dithiothreitol, $1 \times$ complete protease inhibitor cocktail [SigmaAldrich] and 2\% [wt/vol] polyvinylpolypyrrolidone) (Choi et al. 2012) was used to extract total proteins. Sodium dodecyl sulfate-polyacrylamide gel electrophoresis was performed to separate the proteins of different sizes. Rabbit anti-HAperoxidase antibody (Abcam) and goat antirabbit antibody were used to detect the protein level of HA-fused protein. For the ChIP assay, the pepper leaves transiently overexpressing WRKY40-HA, were harvested for chromatin isolation. The isolated chromatins were trimmed with micrococcal nuclease to an average length of 200 to $500 \mathrm{bp}$. Anti-HA antibodies were used to immunoprecipitate the DNA-protein complex of the clipped chromatin. The immunoprecipitated DNA was analyzed for enrichment of WRKY40 in the promoter of ChiIV3 by PCR.

\section{MST analysis.}

Monolith NT. 115 system (Nano Temper) was used to perform MST experiments using 100\% LED and 20\% IR-laser power. Purified WRKY40-6HIS fusion protein was labeled using a monolith NT protein labeling kit RED-NHS (Nano Temper) that reacts with primary amines of targeted protein to form stable dye-protein conjugates. The labeling procedure was performed according to the aforementioned manual. The oligonucleotides of the W4-containing fragment (GAAGTGA TAAGCTGTCAACTAAGTGACGAGCTA) and its mutants (GAAGTGATAAGCTAAGGACTAAGTGACGAGCTA; mutated nucleotides are underlined) were synthesized by Convenience Biology Co., Ltd. and were dissolved in water $(100 \mu \mathrm{M})$. The samples were added into Premium Capillaries and were measured in $20 \mathrm{mM}$ HEPES buffer, pH 7.5, $200 \mathrm{mM} \mathrm{KCl}$, $10 \mathrm{mM}$ TCEP, and $0.05 \%$ Tween 20 . Data were analyzed during acquisition (on the fly data analysis) using NT-Analysis software provided with the system.

\section{Total RNA isolation and real-time PCR analysis.}

Trizol was used for the extraction of total RNA from the pepper leaves. Real-time quantitative reverse transcription (RT)-PCR) was performed with specific primers (Supplementary Table S2), to determine the relative transcriptional levels of targeted genes, following the manufacturer's 
instructions on a Bio-Rad Real-Time PCR system (Bio-Rad) and SYBR Premix Ex Taq II system (Takara). Total RNA isolation and real-time RT-PCR assays were performed following previously described procedures (Cai et al. 2015; Dang et al. 2013; Shen et al. 2016b). Three or six (transient overexpression) independent biological replicates were used for each treatment. Data were analyzed by the Livak method and were expressed as a normalized relative expression level $\left(2^{-\Delta \Delta \mathrm{CT}}\right)$ of the respective genes (Livak and Schmittgen 2001). The relative transcriptional level of each sample was normalized by CaACTIN (GQ339766) and $18 S$ ribosomal RNA (EF564281), respectively.

\section{ACKNOWLEDGMENTS}

We thank M. D. Curtis (University of Zurich) for kindly providing Gateway-destination vectors and S. P. Dinesh-Kumar (Yale University) for providing pTRV1 and pTRV2.

\section{AUTHOR-RECOMMENDED INTERNET RESOURCE}

PlantCARE database:

http://bioinformatics.psb.ugent.be/webtools/plantcare/html

\section{LITERATURE CITED}

Adachi, H., Ishihama, N., Nakano, T., Yoshioka, M., and Yoshioka, H. 2016. Nicotiana benthamiana MAPK-WRKY pathway confers resistance to a necrotrophic pathogen Botrytis cinerea. Plant Signal. Behav. 11: e1183085.

Agarwal, P., Reddy, M. P., and Chikara, J. 2011. WRKY: Its structure, evolutionary relationship, DNA-binding selectivity, role in stress tolerance and development of plants. Mol. Biol. Rep. 38:3883-3896.

Ahmed, N. U., Park, J. I., Jung, H. J., Kang, K. K., Hur, Y., Lim, Y. P., and Nou, I. S. 2012. Molecular characterization of stress resistance-related chitinase genes of Brassica rapa. Plant Physiol. Biochem. 58:106-115.

Baldoni, E., Mattana, M., Locatelli, F., Consonni, R., Cagliani, L. R., Picchi, V., Abbruscato, P., and Genga, A. 2013. Analysis of transcript and metabolite levels in Italian rice (Oryza sativa L.) cultivars subjected to osmotic stress or benzothiadiazole treatment. Plant Physiol. Biochem. 70:492-503.

Baykal, U., Moyne, A. L., and Tuzun, S. 2006. A frameshift in the coding region of a novel tomato class I basic chitinase gene makes it a pseudogene with a functional wound-responsive promoter. Gene 376:37-46.

Brand, L. H., Fischer, N. M., Harter, K., Kohlbacher, O., and Wanke, D. 2013. Elucidating the evolutionary conserved DNA-binding specificities of WRKY transcription factors by molecular dynamics and in vitro binding assays. Nucleic Acids Res. 41:9764-9778.

Cai, H., Yang, S., Yan, Y., Xiao, Z., Cheng, J., Wu, J., Qiu, A., Lai, Y., Mou, S., Guan, D., Huang, R., and He, S. 2015. CaWRKY6 transcriptionally activates CaWRKY40, regulates Ralstonia solanacearum resistance, and confers high-temperature and high-humidity tolerance in pepper. J. Exp. Bot. 66:3163-3174.

Chen, H., Lai, Z., Shi, J., Xiao, Y., Chen, Z., and Xu, X. 2010. Roles of arabidopsis WRKY18, WRKY40 and WRKY60 transcription factors in plant responses to abscisic acid and abiotic stress. BMC Plant Biol. 10:281.

Chen, W., Qu, M., Zhou, Y., and Yang, Q. 2018. Structural analysis of group II chitinase (ChtII) catalysis completes the puzzle of chitin hydrolysis in insects. J. Biol. Chem. 293:2652-2660.

Chen, X. L., Song, R. T., Yu, M. Y., Sui, J. M., Wang, J. S., and Qiao, L. X. 2015. Cloning and functional analysis of the chitinase gene promoter in peanut. Genet. Mol. Res. 14:12710-12722.

Cheng, Y., Zhou, Y., Yang, Y., Chi, Y. J., Zhou, J., Chen, J. Y., Wang, F., Fan, B., Shi, K., Zhou, Y. H., Yu, J. Q., and Chen, Z. 2012. Structural and functional analysis of VQ motif-containing proteins in Arabidopsis as interacting proteins of WRKY transcription factors. Plant Physiol. 159: 810-825.

Choi, D. S., and Hwang, B. K. 2011. Proteomics and functional analyses of pepper abscisic acid-responsive $1(A B R 1)$, which is involved in cell death and defense signaling. Plant Cell 23:823-842.

Choi, D. S., Hwang, I. S., and Hwang, B. K. 2012. Requirement of the cytosolic interaction between PATHOGENESIS-RELATED PROTEIN10 and LEUCINE-RICH REPEAT PROTEIN1 for cell death and defense signaling in pepper. Plant Cell 24:1675-1690.
Choi, H. W., Kim, N. H., Lee, Y. K., and Hwang, B. K. 2013. The pepper extracellular xyloglucan-specific endo- $\beta$-1,4-glucanase inhibitor protein gene, CaXEGIP1, is required for plant cell death and defense responses. Plant Physiol. 161:384-396.

Ciolkowski, I., Wanke, D., Birkenbihl, R. P., and Somssich, I. E. 2008. Studies on DNA-binding selectivity of WRKY transcription factors lend structural clues into WRKY-domain function. Plant Mol. Biol. 68:81-92.

Dang, F., Wang, Y., She, J., Lei, Y., Liu, Z., Eulgem, T., Lai, Y., Lin, J., Yu, L., Lei, D., Guan, D., Li, X., Yuan, Q., and He, S. 2014. Overexpression of CaWRKY27, a subgroup IIe WRKY transcription factor of Capsicum annuиm, positively regulates tobacco resistance to Ralstonia solanacearum infection. Physiol. Plant. 150:397-411.

Dang, F. F., Wang, Y. N., Yu, L., Eulgem, T., Lai, Y., Liu, Z. Q., Wang, X., Qiu, A. L., Zhang, T. X., Lin, J., Chen, Y. S., Guan, D. Y., Cai, H. Y., Mou, S. L., and He, S. L. 2013. CaWRKY40, a WRKY protein of pepper, plays an important role in the regulation of tolerance to heat stress and resistance to Ralstonia solanacearum infection. Plant Cell Environ. 36:757-774

Eulgem, T. 2006. Dissecting the WRKY web of plant defense regulators. PLoS Pathog. 2:e126.

Eulgem, T., Rushton, P. J., Robatzek, S., and Somssich, I. E. 2000. The WRKY superfamily of plant transcription factors. Trends Plant Sci. 5: 199-206.

Eulgem, T., Rushton, P. J., Schmelzer, E., Hahlbrock, K., and Somssich, I. E. 1999. Early nuclear events in plant defence signalling: Rapid gene activation by WRKY transcription factors. EMBO J. 18:4689-4699.

Eulgem, T., and Somssich, I. E. 2007. Networks of WRKY transcription factors in defense signaling. Curr. Opin. Plant Biol. 10:366-371.

Filippi, A., Petrussa, E., Rajcevic, U., Curin Serbec, V., Passamonti, S., Renzone, G., Scaloni, A., Zancani, M., Vianello, A., and Braidot, E. 2016. Flavonoid interaction with a chitinase from grape berry skin: Protein identification and modulation of the enzymatic activity. Molecules 21(10):1300.

Fu, L., Zhu, C., Ding, X., Yang, X., Morris, P. F., Tyler, B. M., and Zhang, X. 2015. Characterization of cell-death-inducing members of the pectate lyase gene family in Phytophthora capsici and their contributions to infection of pepper. Mol. Plant-Microbe Interact 28:766-775.

Gao, X., and He, P. 2013. Nuclear dynamics of Arabidopsis calciumdependent protein kinases in effector-triggered immunity. Plant Signal Behav. 8:e23868.

Gao, Y., Jia, S., Wang, C., Wang, F., Wang, F., and Zhao, K. 2016. BjMYB1, a transcription factor implicated in plant defence through activating BjCHI1 chitinase expression by binding to a W-box-like element. J. Exp. Bot. 67:4647-4658

Gao, Y., Zan, X. L., Wu, X. F., Yao, L., Chen, Y. L., Jia, S. W., and Zhao, K. J. 2014. Identification of fungus-responsive cis-acting element in the promoter of Brassica juncea chitinase gene, BjCHI1. Plant Sci. 215-216: 190-198.

Geilen, K., and Böhmer, M. 2015. Dynamic subnuclear relocalization of WRKY40, a potential new mechanism of ABA-dependent transcription factor regulation. Plant Signal. Behav. 10:e1106659.

Giri, P., Taj, G., Tasleem, M., and Kumar, A. 2013. in silico-prediction of downstream WRKY interacting partners of MAPK3 in Brassica. Bioinformation 9:1036-1039.

Guan, Y., and Chye, M. L. 2008. A Brassica juncea chitinase with two-chitin binding domains show anti-microbial properties against phytopathogens and gram-negative bacteria. Plant Signal. Behav. 3:1103-1105.

Hadwiger, L. A. 2015. Anatomy of a nonhost disease resistance response of pea to Fusarium solani: PR gene elicitation via DNase, chitosan and chromatin alterations. Front. Plant Sci. 6:373.

Hein, I., Gilroy, E. M., Armstrong, M. R., and Birch, P. R. 2009. The zigzag-zig in oomycete-plant interactions. Mol. Plant Pathol. 10:547-562.

Hong, J. K., and Hwang, B. K. 2002. Induction by pathogen, salt and drought of a basic class II chitinase mRNA and its in situ localization in pepper (Capsicum annuum). Physiol. Plant. 114:549-558.

Hong, J. K., and Hwang, B. K. 2006. Promoter activation of pepper class II basic chitinase gene, $C A C h i 2$, and enhanced bacterial disease resistance and osmotic stress tolerance in the CAChi2-overexpressing Arabidopsis. Planta 223:433-448.

Hong, J. K., Jung, H. W., Kim, Y. J., and Hwang, B. K. 2000. Pepper gene encoding a basic class II chitinase is inducible by pathogen and ethephon. Plant Sci. 159:39-49.

Hwang, I. S., An, S. H., and Hwang, B. K. 2011. Pepper asparagine synthetase $1(\mathrm{CaAS} 1)$ is required for plant nitrogen assimilation and defense responses to microbial pathogens. Plant J. 67:749-762.

Iseli, B., Armand, S., Boller, T., Neuhaus, J. M., and Henrissat, B. 1996. Plant chitinases use two different hydrolytic mechanisms. FEBS Lett 382:186-188. 
Ishihama, N., Adachi, H., Yoshioka, M., and Yoshioka, H. 2014. In vivo phosphorylation of WRKY transcription factor by MAPK. Methods Mol. Biol. 1171:171-181.

Ishihama, N., Yamada, R., Yoshioka, M., Katou, S., and Yoshioka, H. 2011. Phosphorylation of the Nicotiana benthamiana WRKY8 transcription factor by MAPK functions in the defense response. Plant Cell 23. 1153-1170.

Ishihama, N., and Yoshioka, H. 2012. Post-translational regulation of WRKY transcription factors in plant immunity. Curr. Opin. Plant Biol. $15: 431-437$.

Jones, J. D., and Dangl, J. L. 2006. The plant immune system. Nature 444: 323-329.

Jones, J. D., Vance, R. E., and Dangl, J. L. 2016. Intracellular innate immune surveillance devices in plants and animals. Science 354: aaf6395.

Karmakar, S., Molla, K. A., Chanda, P. K., Sarkar, S. N., Datta, S. K., and Datta, K. 2016. Green tissue-specific co-expression of chitinase and oxalate oxidase 4 genes in rice for enhanced resistance against sheath blight. Planta 243:115-130.

Kashyap, P., and Deswal, R. 2017. A novel class I Chitinase from Hippophae rhamnoides: Indications for participating in ICE-CBF cold stress signaling pathway. Plant Sci. 259:62-70.

Khan, M. S., Sadat, S. U., Jan, A., and Munir, I. 2017. Impact of transgenic Brassica napus harboring the antifungal synthetic chitinase $(\mathrm{NiC})$ gene on rhizosphere microbial diversity and enzyme activities. Front. Plant Sci. 8:1307.

Kim, C. Y., and Zhang, S. 2004. Activation of a mitogen-activated protein kinase cascade induces WRKY family of transcription factors and defense genes in tobacco. Plant J. 38:142-151.

Kim, D. S., and Hwang, B. K. 2011. The pepper receptor-like cytoplasmic protein kinase CaPIK1 is involved in plant signaling of defense and celldeath responses. Plant J. 66:642-655.

Kim, D. S., Kim, N. H., and Hwang, B. K. 2015. The Capsicum annuum class IV chitinase ChitIV interacts with receptor-like cytoplasmic protein kinase PIK1 to accelerate PIK1-triggered cell death and defence responses. J. Exp. Bot. 66:1987-1999.

Kim, H. J., Choi, H. S., Yang, S. Y., Kim, I. S., Yamaguchi, T., Sohng, J. K., Park, S. K., Kim, J. C., Lee, C. H., Gardener, B. M., and Kim, Y. C. 2014a. Both extracellular chitinase and a new cyclic lipopeptide, chromobactomycin, contribute to the biocontrol activity of Chromobacterium sp. C61. Mol. Plant Pathol. 15:122-132.

Kim, K. C., Lai, Z., Fan, B., and Chen, Z. 2008. Arabidopsis WRKY38 and WRKY62 transcription factors interact with histone deacetylase 19 in basal defense. Plant Cell 20:2357-2371.

Kim, S., Park, M., Yeom, S. I., Kim, Y. M., Lee, J. M., Lee, H. A., Seo, E., Choi, J., Cheong, K., Kim, K. T., Jung, K., Lee, G. W., Oh, S. K., Bae, C., Kim, S. B., Lee, H. Y., Kim, S. Y., Kim, M. S., Kang, B. C., Jo, Y. D., Yang, H. B., Jeong, H. J., Kang, W. H., Kwon, J. K., Shin, C., Lim, J. Y., Park, J. H., Huh, J. H., Kim, J. S., Kim, B. D., Cohen, O., Paran, I., Suh, M. C., Lee, S. B., Kim, Y. K., Shin, Y., Noh, S. J., Park, J., Seo, Y. S., Kwon, S. Y., Kim, H. A., Park, J. M., Kim, H. J., Choi, S. B., Bosland, P. W., Reeves, G., Jo, S. H., Lee, B. W., Cho, H. T., Choi, H. S., Lee, M. S., Yu, Y., Do Choi, Y., Park, B. S., van Deynze, A., Ashrafi, H., Hill, T., Kim, W. T., Pai, H. S., Ahn, H. K., Yeam, I., Giovannoni, J. J., Rose, J. K., Sørensen, I., Lee, S. J., Kim, R. W., Choi, I. Y., Choi, B. S., Lim, J. S., Lee, Y. H., and Choi, D. 2014b. Genome sequence of the hot pepper provides insights into the evolution of pungency in Capsicum species. Nat. Genet. 46:270-278.

Kim, S. Y., Chung, H. J., and Thomas, T. L. 1997. Isolation of a novel class of bZIP transcription factors that interact with ABA-responsive and embryo-specification elements in the Dc3 promoter using a modified yeast one-hybrid system. Plant J. 11:1237-1251.

Lee, S. Y., Tindwa, H., Lee, Y. S., Naing, K. W., Hong, S. H., Nam, Y., and Kim, K. Y. 2012. Biocontrol of anthracnose in pepper using chitinase, $\beta$-1,3 glucanase, and 2-furancarboxaldehyde produced by Streptomyces cavourensis SY224. J. Microbiol. Biotechnol. 22:1359-1366.

Lei, R., Li, X., Ma, Z., Lv, Y., Hu, Y., and Yu, D. 2017. Arabidopsis WRKY2 and WRKY34 transcription factors interact with VQ20 protein to modulate pollen development and function. Plant J. 91: 962-976.

Li, Z. T., Dhekney, S. A., and Gray, D. J. 2011. PR-1 gene family of grapevine: A uniquely duplicated PR-1 gene from a Vitis interspecific hybrid confers high level resistance to bacterial disease in transgenic tobacco. Plant Cell Rep. 30:1-11.

Liu, Y., Schiff, M., and Dinesh-Kumar, S. P. 2004. Involvement of MEK1 MAPKK, NTF6 MAPK, WRKY/MYB transcription factors, COI1 and CTR1 in N-mediated resistance to tobacco mosaic virus. Plant J. 38: 800-809.
Liu, Z., Shi, L., Yang, S., Lin, Y., Weng, Y., Li, X., Hussain, A., Noman, A., and He, S. 2017. Functional and promoter analysis of ChiIV3, a chitinase of pepper plant, in response to Phytophthora capsici infection. Int. J. Mol. Sci. 18:1661.

Liu, Z. Q., Liu, Y. Y., Shi, L. P., Yang, S., Shen, L., Yu, H. X., Wang, R. Z., Wen, J. Y., Tang, Q., Hussain, A., Khan, M. I., Hu, J., Liu, C. L., Zhang, Y. W., Cheng, W., and He, S. L. 2016. SGT1 is required in PcINF1/SRC2-1 induced pepper defense response by interacting with SRC2-1. Sci. Rep. 6: 21651.

Liu, Z. Q., Qiu, A. L., Shi, L. P., Cai, J. S., Huang, X. Y., Yang, S., Wang, B., Shen, L., Huang, M. K., Mou, S. L., Ma, X. L., Liu, Y. Y., Lin, L., Wen, J. Y., Tang, Q., Shi, W., Guan, D. Y., Lai, Y., and He, S. L. 2015. SRC2-1 is required in PcINF1-induced pepper immunity by acting as an interacting partner of PcINF1. J. Exp. Bot. 66:3683-3698.

Liu, Z. Q., Yan, L., Wu, Z., Mei, C., Lu, K., Yu, Y. T., Liang, S., Zhang, X. F., Wang, X. F., and Zhang, D. P. 2012. Cooperation of three WRKYdomain transcription factors WRKY18, WRKY40, and WRKY60 in repressing two ABA-responsive genes ABI4 and ABI5 in Arabidopsis. J. Exp. Bot. 63:6371-6392.

Livak, K. J., and Schmittgen, T. D. 2001. Analysis of relative gene expression data using real-time quantitative PCR and the $2^{-\Delta \Delta C T}$ method. Methods 25:402-408.

Maximova, S. N., Marelli, J. P., Young, A., Pishak, S., Verica, J. A., and Guiltinan, M. J. 2006. Over-expression of a cacao class I chitinase gene in Theobroma cacao L. enhances resistance against the pathogen, Colletotrichum gloeosporioides. Planta 224:740-749.

Mészáros, P., Rybanský, L., Spieß, N., Socha, P., Kuna, R., Libantová, J., Moravčíková, J., Piršelová, B., Hauptvogel, P., and Matušíková, I. 2014 Plant chitinase responses to different metal-type stresses reveal specificity. Plant Cell Rep. 33:1789-1799.

Miao, Y., Laun, T. M., Smykowski, A., and Zentgraf, U. 2007. Arabidopsis MEKK1 can take a short cut: It can directly interact with senescencerelated WRKY53 transcription factor on the protein level and can bind to its promoter. Plant Mol. Biol. 65:63-76.

Miao, Y., and Zentgraf, U. 2010. A HECT E3 ubiquitin ligase negatively regulates Arabidopsis leaf senescence through degradation of the transcription factor WRKY53. Plant J. 63:179-188.

Mishra, P., Kshirsagar, P. R., Nilegaonkar, S. S., and Singh, S. K. 2012 Statistical optimization of medium components for production of extracellular chitinase by Basidiobolus ranarum: A novel biocontrol agent against plant pathogenic fungi. J. Basic Microbiol. 52:539-548.

Miyamoto, K., Shimizu, T., Lin, F., Sainsbury, F., Thuenemann, E., Lomonossoff, G., Nojiri, H., Yamane, H., and Okada, K. 2012. Identification of an E-box motif responsible for the expression of jasmonic acid-induced chitinase gene OsChia4a in rice. J. Plant Physiol. 169:621-627.

Mou, S., Liu, Z., Guan, D., Qiu, A., Lai, Y., and He, S. 2013. Functional analysis and expressional characterization of rice ankyrin repeatcontaining protein, OsPIANK1, in basal defense against Magnaporthe oryzae attack. PLoS One 8:e59699.

Mur, L. A. J., Simpson, C., Kumari, A., Gupta, A. K., and Gupta, K. J. 2017. Moving nitrogen to the centre of plant defence against pathogens. Ann Bot. 119:703-709.

Murray, S. L., Adams, N., Kliebenstein, D. J., Loake, G. J., and Denby, K. J. 2005. A constitutive PR-1:luciferase expression screen identifies Arabidopsis mutants with differential disease resistance to both biotrophic and necrotrophic pathogens. Mol. Plant Pathol. 6:31-41.

Nakahara, K. S., and Masuta, C. 2014. Interaction between viral RNA silencing suppressors and host factors in plant immunity. Curr. Opin. Plant Biol. 20:88-95.

Pandey, S. P., and Somssich, I. E. 2009. The role of WRKY transcription factors in plant immunity. Plant Physiol. 150:1648-1655.

Park, C. Y., Lee, J. H., Yoo, J. H., Moon, B. C., Choi, M. S., Kang, Y. H., Lee, S. M., Kim, H. S., Kang, K. Y., Chung, W. S., Lim, C. O., and Cho, M. J. 2005. WRKY group IId transcription factors interact with calmodulin. FEBS Lett. 579:1545-1550.

Park, H. J., Kim, S. J., Park, S. J., Eom, S. H., Gu, G. J., Kim, S. H., and Youn, H. S. 2013. Phenethyl isothiocyanate regulates inflammation through suppression of the TRIF-dependent signaling pathway of Tolllike receptors. Life Sci. 92:793-798.

Pecher, P., Eschen-Lippold, L., Herklotz, S., Kuhle, K., Naumann, K., Bethke, G., Uhrig, J., Weyhe, M., Scheel, D., and Lee, J. 2014. The Arabidopsis thaliana mitogen-activated protein kinases MPK3 and MPK6 target a subclass of 'VQ-motif'-containing proteins to regulate immune responses. New Phytol. 203:592-606.

Qiu, A., Lei, Y., Yang, S., Wu, J., Li, J., Bao, B., Cai, Y., Wang, S., Lin, J., Wang, Y., Shen, L., Cai, J., Guan, D., and He, S. 2018. CaC3H14 encoding a tandem $\mathrm{CCCH}$ zinc finger protein is directly targeted by CaWRKY40 and positively regulates the response of pepper to 
inoculation by Ralstonia solanacearum. Mol. Plant Pathol. 19: 2221-2235.

Richa, K., Tiwari, I. M., Kumari, M., Devanna, B. N., Sonah, H., Kumari, A., Nagar, R., Sharma, V., Botella, J. R., and Sharma, T. R. 2016. functional characterization of novel chitinase genes present in the sheath blight resistance QTL: qSBR11-1 in rice line Tetep. Front. Plant Sci. 7: 244

Römer, P., Hahn, S., Jordan, T., Strauss, T., Bonas, U., and Lahaye, T. 2007. Plant pathogen recognition mediated by promoter activation of the pepper Bs3 resistance gene. Science 318:645-648.

Rushton, D. L., Tripathi, P., Rabara, R. C., Lin, J., Ringler, P., Boken, A. K. Langum, T. J., Smidt, L., Boomsma, D. D., Emme, N. J., Chen, X., Finer, J. J., Shen, Q. J., and Rushton, P. J. 2012. WRKY transcription factors: Key components in abscisic acid signalling. Plant Biotechnol. J. 10:2-11.

Rushton, P. J., Somssich, I. E., Ringler, P., and Shen, Q. J. 2010. WRKY transcription factors. Trends Plant Sci. 15:247-258.

Shen, H., Liu, C., Zhang, Y., Meng, X., Zhou, X., Chu, C., and Wang, X. 2012. OsWRKY30 is activated by MAP kinases to confer drought tolerance in rice. Plant Mol. Biol. 80:241-253.

Shen, L., Liu, Z., Yang, S., Yang, T., Liang, J., Wen, J., Liu, Y., Li, J., Shi, L., Tang, Q., Shi, W., Hu, J., Liu, C., Zhang, Y., Lin, W., Wang, R., Yu, H., Mou, S., Hussain, A., Cheng, W., Cai, H., He, L., Guan, D., Wu, Y., and He, S. 2016b. Pepper CabZIP63 acts as a positive regulator during Ralstonia solanacearum or high temperature-high humidity challenge in a positive feedback loop with CaWRKY40. J. Exp. Bot. 67:2439-2451.

Shen, L., Yang, S., Yang, T., Liang, J., Cheng, W., Wen, J., Liu, Y., Li, J., Shi, L., Tang, Q., Shi, W., Hu, J., Liu, C., Zhang, Y., Mou, S., Liu, Z., Cai, H., He, L., Guan, D., Wu, Y., and He, S. 2016a. CaCDPK15 positively regulates pepper responses to Ralstonia solanacearum inoculation and forms a positive-feedback loop with CaWRKY40 to amplify defense signaling. Sci. Rep. 6:22439.

Singh, V., Banday, Z. Z., and Nandi, A. K. 2014a. Exogenous application of histone demethylase inhibitor trans-2-phenylcyclopropylamine mimics FLD loss-of-function phenotype in terms of systemic acquired resistance in Arabidopsis thaliana. Plant Signal. Behav. 9:e29658.

Singh, V., Roy, S., Singh, D., and Nandi, A. K. 2014b. Arabidopsis flowering locus D influences systemic-acquired-resistance- induced expression and histone modifications of WRKY genes. J. Biosci. 39:119-126.

Tang, W., Newton, R. J., Li, C., and Charles, T. M. 2007. Enhanced stress tolerance in transgenic pine expressing the pepper $\mathrm{CaPFl}$ gene is associated with the polyamine biosynthesis. Plant Cell Rep. 26:115-124.

Thomma, B. P., Nürnberger, T., and Joosten, M. H. 2011. Of PAMPs and effectors: The blurred PTI-ETI dichotomy. Plant Cell 23:4-15.

Tobias, D. J., Manoharan, M., Pritsch, C., and Dahleen, L. S. 2007. Cobombardment, integration and expression of rice chitinase and thaumatin-like protein genes in barley (Hordeum vulgare cv. Conlon). Plant Cell Rep. 26:631-639.

Tominaga, J., Mizutani, H., Horikawa, D., Nakahara, Y., Takami, T., Sakamoto, W., Sakamoto, A., and Shimada, H. 2016. Rice CYO1, an ortholog of Arabidopsis thaliana cotyledon chloroplast biogenesis factor AtCYO1, is expressed in leaves and involved in photosynthetic performance. J. Plant Physiol. 207:78-83.

Tsuda, K., and Katagiri, F. 2010. Comparing signaling mechanisms engaged in pattern-triggered and effector-triggered immunity. Curr. Opin. Plant Biol. 13:459-465.

Tsuda, K., Mine, A., Bethke, G., Igarashi, D., Botanga, C. J., Tsuda, Y., Glazebrook, J., Sato, M., and Katagiri, F. 2013. Dual regulation of gene expression mediated by extended MAPK activation and salicylic acid contributes to robust innate immunity in Arabidopsis thaliana. PLoS Genet. 9:e1004015.

van Elsas, J. D., Kastelein, P., de Vries, P. M., and van Overbeek, L. S. 2001. Effects of ecological factors on the survival and physiology of Ralstonia solanacearum bv. 2 in irrigation water. Can. J. Microbiol. 47:842-854.

Vanholme, B., Vanholme, R., Turumtay, H., Goeminne, G., Cesarino, I., Goubet, F., Morreel, K., Rencoret, J., Bulone, V., Hooijmaijers, C., De Rycke, R., Gheysen, G., Ralph, J., De Block, M., Meulewaeter, F., and Boerjan, W. 2014. Accumulation of $N$-acetylglucosamine oligomers in the plant cell wall affects plant architecture in a dose-dependent and conditional manner. Plant Physiol. 165:290-308.

Vasavirama, K., and Kirti, P. B. 2012. Increased resistance to late leaf spot disease in transgenic peanut using a combination of PR genes. Funct. Integr. Genomics 12:625-634.
Wan, J., Zhang, X. C., Neece, D., Ramonell, K. M., Clough, S., Kim, S. Y., Stacey, M. G., and Stacey, G. 2008b. A LysM receptor-like kinase plays a critical role in chitin signaling and fungal resistance in Arabidopsis. Plant Cell 20:471-481.

Wan, J., Zhang, X. C., and Stacey, G. 2008a. Chitin signaling and plant disease resistance. Plant Signal. Behav. 3:831-833.

Wang, M., Vannozzi, A., Wang, G., Zhong, Y., Corso, M., Cavallini, E., and Cheng, Z. M. 2015. A comprehensive survey of the grapevine VQ gene family and its transcriptional correlation with WRKY proteins. Front. Plant Sci. 6:417.

Wang, Q., Ma, Y., Yang, H., and Chang, Z. 2014. Effect of biofumigation and chemical fumigation on soil microbial community structure and control of pepper Phytophthora blight. World J. Microbiol. Biotechnol. 30:507-518

Weyhe, M., Eschen-Lippold, L., Pecher, P., Scheel, D., and Lee, J. 2014a. Ménage à trois: The complex relationships between mitogen-activated protein kinases, WRKY transcription factors, and VQ-motif-containing proteins. Plant Signal. Behav. 9:e29519.

Wu, J., Wang, Y., Kim, S. G., Jung, K. H., Gupta, R., Kim, J., Park, Y., Kang, K. Y., and Kim, S. T. 2017. A secreted chitinase-like protein (OsCLP) supports root growth through calcium signaling in Oryza sativa. Physiol. Plant. 161:273-284.

Xu, X., Chen, C., Fan, B., and Chen, Z. 2006. Physical and functional interactions between pathogen-induced Arabidopsis WRKY18, WRKY40, and WRKY60 transcription factors. Plant Cell 18:1310-1326.

Yamasaki, K., Kigawa, T., Inoue, M., Tateno, M., Yamasaki, T., Yabuki, T., Aoki, M., Seki, E., Matsuda, T., Tomo, Y., Hayami, N., Terada, T., Shirouzu, M., Tanaka, A., Seki, M., Shinozaki, K., and Yokoyama, S. 2005. Solution structure of an Arabidopsis WRKY DNA binding domain. Plant Cell 17:944-956.

Yamasaki, K., Kigawa, T., Watanabe, S., Inoue, M., Yamasaki, T., Seki, M., Shinozaki, K., and Yokoyama, S. 2012. Structural basis for sequencespecific DNA recognition by an Arabidopsis WRKY transcription factor. J. Biol. Chem. 287:7683-7691.

Yang, W., Zhang, H., Li, M., Wang, Z., Zhou, J., Wang, S., Lu, G., and Fu, F. 2014. Early diagnosis of blast fungus, Magnaporthe oryzae, in rice plant by using an ultra-sensitive electrically magnetic-controllable electrochemical biosensor. Anal. Chim. Acta 850:85-91.

Yin, Y. X., Wang, S. B., Zhang, H. X., Xiao, H. J., Jin, J. H., Ji, J. J., Jing, H., Chen, R. G., Arisha, M. H., and Gong, Z. H. 2015. Cloning and expression analysis of CaPIP1-1 gene in pepper (Capsicum annuиm L.) Gene 563:87-93

Yoo, S. J., Kim, S. H., Kim, M. J., Ryu, C. M., Kim, Y. C., Cho, B. H., and Yang, K. Y. 2014. Involvement of the OsMKK4-OsMPK1 cascade and its downstream transcription factor OsWRKY53 in the wounding response in rice. Plant Pathol. J. 30:168-177.

Zhang, H., Li, D., Wang, M., Liu, J., Teng, W., Cheng, B., Huang, Q., Wang, M., Song, W., Dong, S., Zheng, X., and Zhang, Z. 2012. The Nicotiana benthamiana mitogen-activated protein kinase cascade and WRKY transcription factor participate in $\mathrm{Nep} 1_{\mathrm{Mo}_{\mathrm{o}}}$-triggered plant responses. Mol. Plant-Microbe Interact 25:1639-1653.

Zhang, X., and Mou, Z. 2009. Extracellular pyridine nucleotides induce $P R$ gene expression and disease resistance in Arabidopsis. Plant J. 57: 302-312.

Zhang, Y., and Feng, J. C. 2014. Identification and characterization of the grape WRKY family. BioMed Res. Int. 2014:787680.

Zhao, G., Ihuegbu, N., Lee, M., Schriefer, L., Wang, T., and Stormo, G. D. 2012. Conserved motifs and prediction of regulatory modules in Caenorhabditis elegans. G3 (Bethesda) 2:469-481.

Zhao, G., Schriefer, L. A., and Stormo, G. D. 2007. Identification of muscle-specific regulatory modules in Caenorhabditis elegans. Genome Res. 17:348-357.

Zhong, Z., Chen, M., Lin, L., Han, Y., Bao, J., Tang, W., Lin, L., Lin, Y., Somai, R., Lu, L., Zhang, W., Chen, J., Hong, Y., Chen, X., Wang, B., Shen, W. C., Lu, G., Norvienyeku, J., Ebbole, D. J., and Wang, Z. 2018. Population genomic analysis of the rice blast fungus reveals specific events associated with expansion of three main clades. ISME J. 12 1867-1878.

Zhou, T., Dagdas, Y. F., Zhu, X., Zheng, S., Chen, L., Cartwright, Z., Talbot, N. J., and Wang, Z. 2017. The glycogen synthase kinase MoGsk1, regulated by Mps1 MAP kinase, is required for fungal development and pathogenicity in Magnaporthe oryzae. Sci. Rep. 7:945. 